\title{
Article \\ Age Dependent Epidemic Modeling of COVID-19 Outbreak in Kuwait, France, and Cameroon
}

\author{
Kayode Oshinubi $^{1}\left(\mathbb{D}\right.$, Sana S. Buhamra ${ }^{2, *}$, Noriah M. Al-Kandari ${ }^{3}$, Jules Waku ${ }^{4}$, Mustapha Rachdi ${ }^{1}$ (D) \\ and Jacques Demongeot ${ }^{1}$ (D)
}

check for updates

Citation: Oshinubi, K.; Buhamra, S.S.; Al-Kandari, N.M.; Waku, J.; Rachdi, M.; Demongeot, J. Age Dependent Epidemic Modeling of COVID-19 Outbreak in Kuwait, France, and Cameroon. Healthcare 2022, 10, 482. https://doi.org/10.3390/ healthcare10030482

Academic Editor: Pedram Sendi

Received: 11 January 2022

Accepted: 28 February 2022

Published: 4 March 2022

Publisher's Note: MDPI stays neutral with regard to jurisdictional claims in published maps and institutional affiliations.

Copyright: (C) 2022 by the authors. Licensee MDPI, Basel, Switzerland. This article is an open access article distributed under the terms and conditions of the Creative Commons Attribution (CC BY) license (https:// creativecommons.org/licenses/by/ $4.0 /)$.
1 Laboratory AGEIS EA 7407, Team Tools for e-Gnosis Medical, Faculty of Medicine, University Grenoble Alpes (UGA), 38700 La Tronche, France; kayode.oshinubi@univ-grenoble-alpes.fr (K.O.); mustapha.rachdi@univ-grenoble-alpes.fr (M.R.); jacques.demongeot@univ-grenoble-alpes.fr (J.D.)

2 Department of Information Science, Kuwait University, P.O. Box 5969, Safat 13060, Kuwait

3 Department of Statistics and Operations Research, Kuwait University, P.O. Box 5969, Safat 13060, Kuwait; noriah.alkandari@ku.edu.kw

4 UMMISCO UMI IRD 209 \& LIRIMA, University of Yaoundé I, Yaoundé P.O. Box 337, Cameroon; jules.waku@facsciences-uy1.cm

* Correspondence: sana.buhamra@ku.edu.kw

\begin{abstract}
Revisiting the classical model by Ross and Kermack-McKendrick, the Susceptible-InfectiousRecovered (SIR) model used to formalize the COVID-19 epidemic, requires improvements which will be the subject of this article. The heterogeneity in the age of the populations concerned leads to considering models in age groups with specific susceptibilities, which makes the prediction problem more difficult. Basically, there are three age groups of interest which are, respectively, 0-19 years, 20-64 years, and >64 years, but in this article, we only consider two (20-64 years and $>64$ years) age groups because the group 0-19 years is widely seen as being less infected by the virus since this age group had a low infection rate throughout the pandemic era of this study, especially the countries under consideration. In this article, we proposed a new mathematical age-dependent (Susceptible-Infectious-Goneanewsusceptible-Recovered (SIGR)) model for the COVID-19 outbreak and performed some mathematical analyses by showing the positivity, boundedness, stability, existence, and uniqueness of the solution. We performed numerical simulations of the model with parameters from Kuwait, France, and Cameroon. We discuss the role of these different parameters used in the model; namely, vaccination on the epidemic dynamics. We open a new perspective of improving an age-dependent model and its application to observed data and parameters.
\end{abstract}

Keywords: SIR model; COVID-19; age-dependent modeling; demographic model; epidemic model

\section{Introduction}

\subsection{Background}

The influence of the median age of a country on the incidence of COVID-19 has been highlighted on numerous occasions, in particular through the appearance of many asymptomatic cases in the age groups below 50. For example, on the site of Johns Hopkins University dedicated to COVID-19 [1], data clearly shows this influence on the case fatality rate corresponding to the cumulative deaths recorded 5 months after the beginning of the outbreak (12 May 2020) vs. the median age of many countries in 2017 (Figure 1). This first observation has been confirmed by many studies in different countries [2-22] such as in France (Figure 2), and we will confirm in this paper that the age of the patients suffering from COVID-19 is a good predictor of severity.

This severity among different age groups increased COVID-19 incidence at the start of the pandemic in terms of hospitalization cases, intensive care unit (ICU) cases, prompting many countries to quickly implement intervention measures such as increasing the number of beds and ventilators in their various hospitals and setting up new isolation 
centers in order to mitigate its spread and save lives, particularly the elderly, who were disproportionately among those who were highly susceptible.

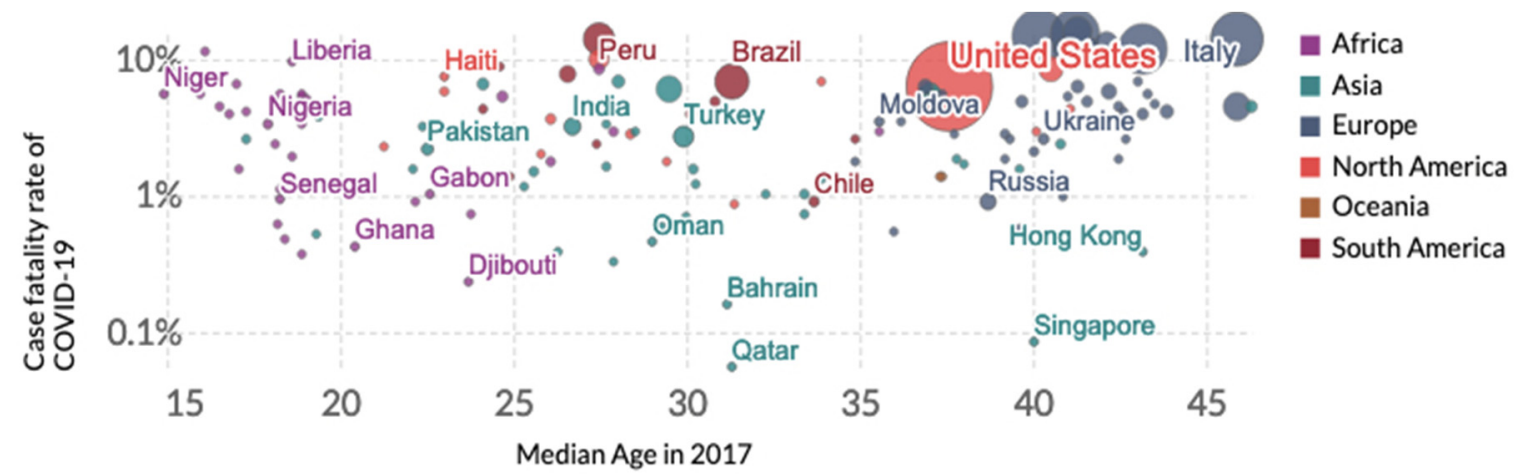

Figure 1. Dependence of the case fatality rate (from cumulative deaths on the 20 May 2020) vs. median age of several countries in 2017 (from [1]). The area of a country circle is proportional to the number of cumulated deaths due to COVID-19 on the 20 May 2020, e.g., for the USA: 99,643 (in red).
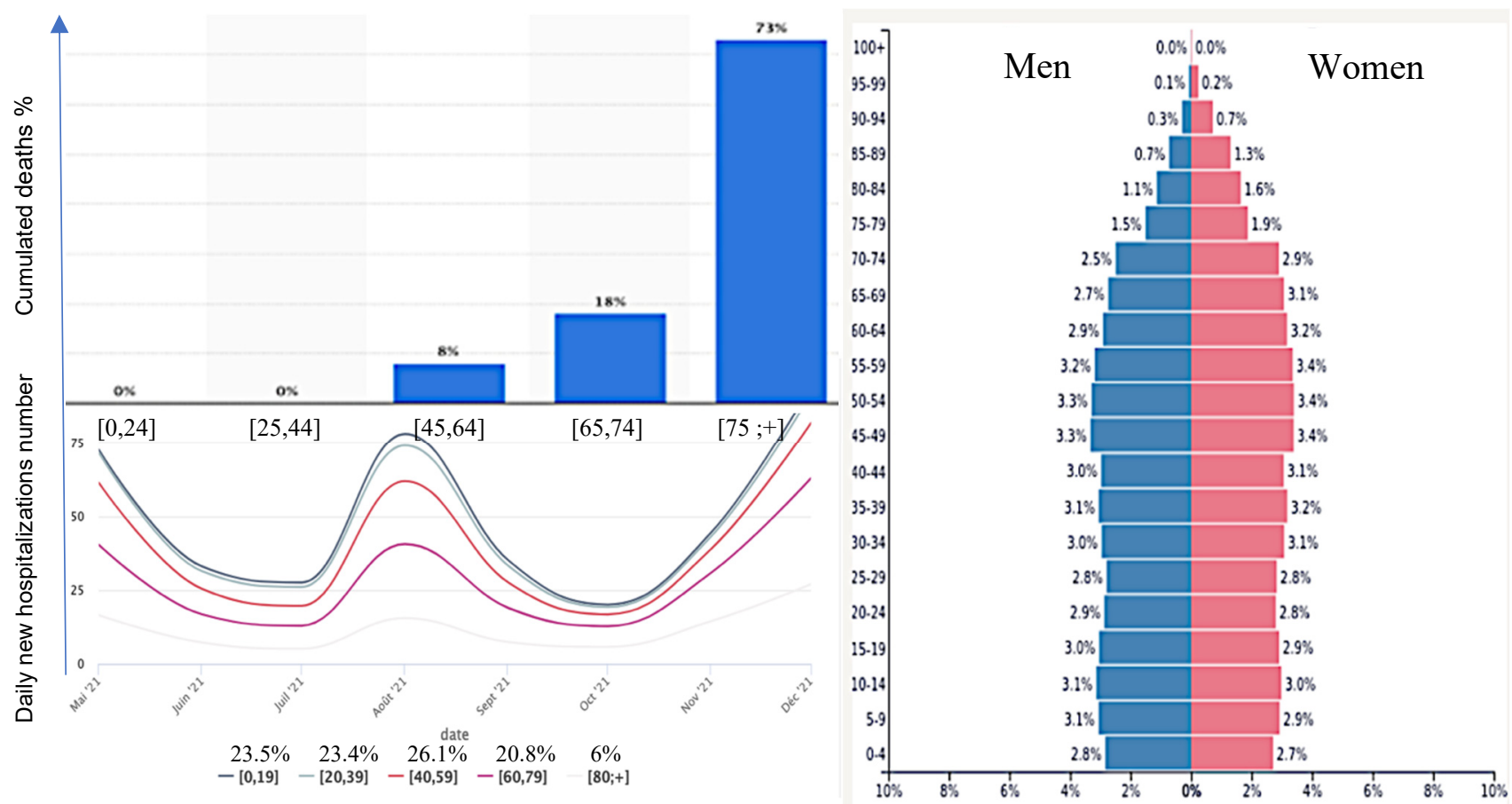

Figure 2. Top left: COVID-19 percentage of death in France by age class [23]. Bottom left: Influence of age (curves with color coding) on COVID-19 hospitalizations in France in the extreme age classes [23]. Bottom right: age classes pyramid in 2020 in France (total population size: 65,273,512) [24].

\subsection{Related Work}

The heterogeneity in the age of the populations studied here (Kuwait, France, and Cameroon) leads to considering a model in age groups with specific susceptibilities for each age class, which makes the prediction problem of the new infectious growth more difficult. Basically, there are three age groups of interest in the COVID-19 outbreak, which are, respectively, 0-19 years, 20-64 years, and >64 years, but here we only consider two (20-64 years and $>64$ years) age groups because the group 0-19 years is widely seen as being less infectious by SARS-CoV-2 [21,22] since this age group had a low infection rate throughout the period considered in this study, especially the countries data used for simulation. 
The heterogeneity in individual age and the reaction to biological and environmental changes that have been observed in COVID-19 dynamics in terms of different reactions to vaccination by age group, severity of infection per age group, hospitalization, and intensive care unit (ICU) records show different patterns, which is why it is important to improve mathematical models for COVID-19 pandemic prediction to account for different proportions of ages in the population, which is a major factor in epidemic history [25]. In some age classes (namely young and adult classes), all infectious are neither symptomatic nor reported and some models incorporating asymptomatic [26], unreported [27], and super-spreaders [28] have been proposed for the COVID-19 outbreak. Many authors have also included age in their models as a gravity factor [29-33], but here, the originality is to propose an improvement of the classical Ross and Kermack-McKendrick model and use it for giving a theoretical and numerical framework for interpreting the relationship between demographic parameters such as age pyramid, fertility and mortality, and epidemiological parameters such as the basic reproduction number $\mathrm{R}_{0}$ and vaccination rate.

In subsequent sections, we present (i) in Section 2, the materials and methods, (ii) in Section 3, the formulation of a new age-dependent dynamical model for the spread of COVID-19, (ii) in Section 4, we provide some mathematical analyses of the model, (iii) in Section 5, we carry out numerical simulations for some COVID-19 parameters from Kuwait, France, and Cameroon; lastly (iv), in Sections 6 and 7, we provide a discussion and some conclusions.

\section{Material and Methods}

\subsection{Material}

The data material is from public epidemiologic and demographic databases $[23,24]$ and the reference methods are both from classical demographic (such as Leslie) and epidemiologic (such as Ross and Kermack-McKendrick) models. In Figures 3 and 4, we present visualizations for pandemic dynamics in different age groups and sexes for Kuwait and Cameroon, respectively, to support the motivation for this article that pandemic evolution and severity are related to age classes, and thus modeling is important as various researchers and health experts are investigating SAR-CoV-2 mutations. The countries under consideration have a higher proportion of young people, while the elderly have a lower proportion. Only $2 \%$ of the total population in Kuwait is over 65 and vulnerable to the pandemic, whereas $20.8 \%$ of the population in France is over 65 and vulnerable to the pandemic, and $2.7 \%$ of the population in Cameroon is over 65 and vulnerable to the pandemic.
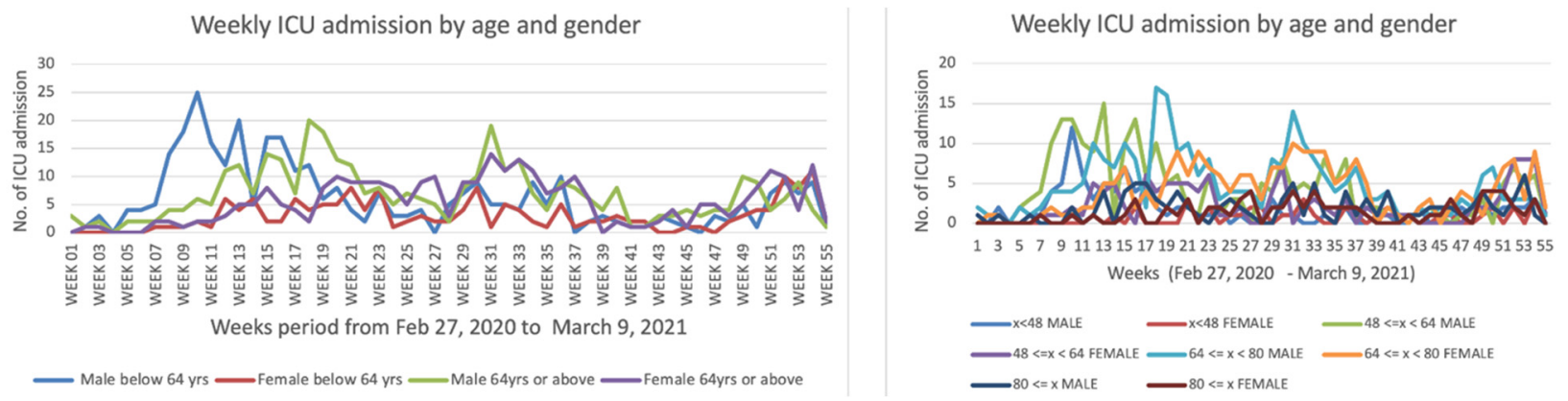

Figure 3. Curves of COVID-19 weekly ICU admissions in Kuwait by age classes (two on the left and four on the right) and gender from 27 February 2020 to 9 March 2021.

\subsection{Leslie Model}

The first population growth model was proposed by Leslie in 1945 using the age pyramid vector $p(t)=\left(p_{j}(t)\right)_{j=1,2, \ldots, m}$, where $p_{j}(t)$ represents the size of the age class $j$ 
at time $t$, with $\mathrm{j}$ ranging from the birth age 1 to the maximal death age $\mathrm{m}$, whose discrete dynamics is governed by the matrix equation given as:

$$
p(t)=L p(t-1) \text { with } L=\left(l_{j k}\right)=\left[\begin{array}{cccccc}
f_{1} & f_{2} & f_{3} & \ldots & \ldots & f_{m} \\
b_{1} & 0 & 0 & \ldots & \ldots & 0 \\
0 & b_{2} & 0 & \ldots & \ldots & 0 \\
\vdots & \vdots & \ddots & \ldots & \ldots & \vdots \\
\vdots & \vdots & \vdots & \ddots & \ldots & \vdots \\
0 & 0 & 0 & \ldots & b_{m-1} & 0
\end{array}\right]
$$

where $\forall \mathrm{j}=1, \ldots, \mathrm{m}, \mathrm{b}_{\mathrm{j}}=1-\mu_{\mathrm{j}} \leq 1$ (where $\mu_{\mathrm{j}}$ is the death rate at age $\mathrm{j}$ ) is the survival probability between ages $j$ and $j+1$ and $f_{1}$ is the birth rate at age $j$ (i.e., the mean number of offsprings from an individual at age $j$ ). The dynamic stability of the Leslie system for a distance $d$ is quantified by the tendency to return to its stationary pyramid $p^{*}$ after a perturbation $\mathrm{p}^{*}+\mathrm{q}$, such as $\mathrm{d}\left(\mathrm{p}^{*}, \mathrm{~L}^{\mathrm{m}}\left(\mathrm{p}^{*}+\mathrm{q}\right)\right)<\mathrm{Ke} \mathrm{e}^{-\mathrm{mD}}$ tends exponentially to 0 , when $\mathrm{m}$ goes to infinity, the parameter $\mathrm{D}$ being the stability module. For the $L^{2}$ distance to the stationary pyramid $\mathrm{p}^{*}, \mathrm{D}=\left|\lambda-\lambda^{\prime}\right|$, absolute value of the difference between the dominant and sub-dominant eigenvalues of $L$, i.e., $\lambda$ and $\lambda^{\prime}\left(\lambda=\mathrm{e}^{\mathrm{r}}\right.$, where $\mathrm{r}$ is the Malthusian growth rate, and $\mathrm{p}^{*}$ is the left eigenvector of L corresponding to $\lambda$ ). For the distance (known as symmetrized divergence) of Kullback-Leibler, $\mathrm{D}=\mathrm{kH}$, where $\mathrm{H}$ is the entropy of $\mathrm{p}^{*}$ and $\mathrm{k}$ is a constant [35-46].
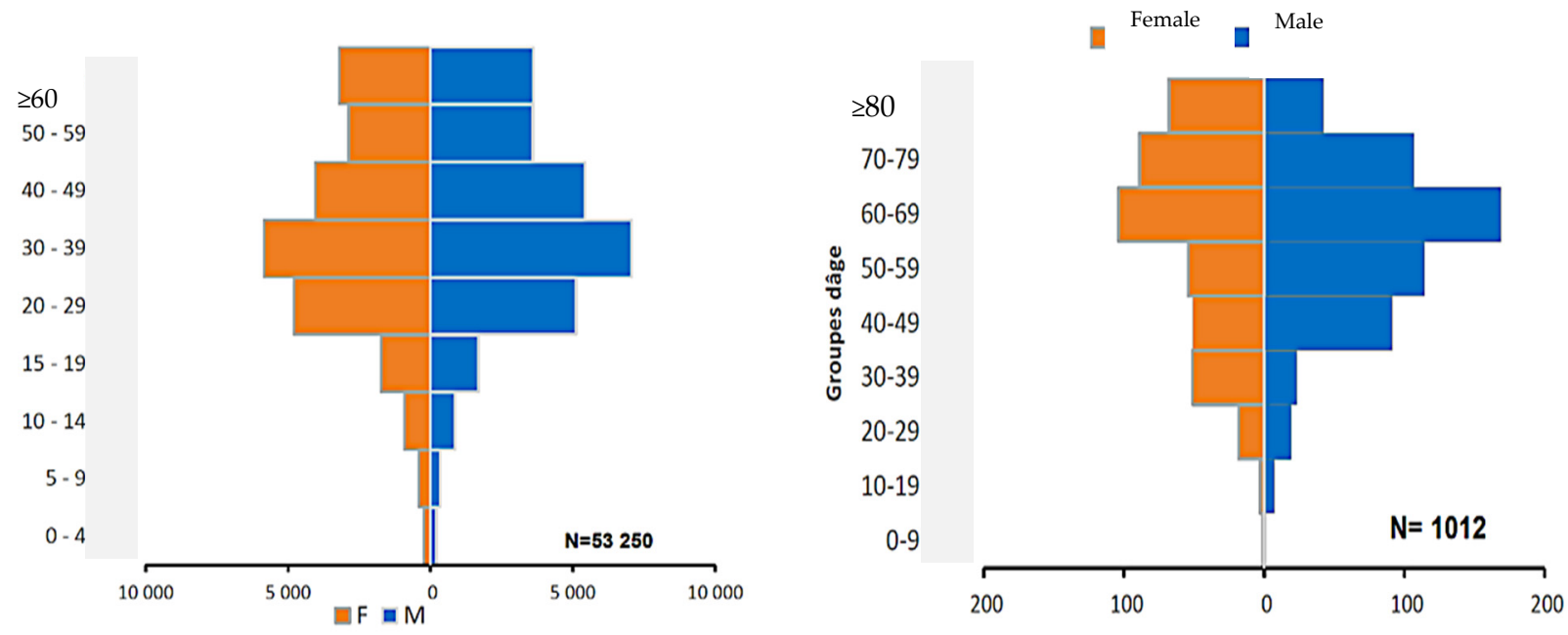

Figure 4. Left: Distribution of cumulated confirmed cases of COVID-19 by age group and gender in Cameroon as of 23 June 2021 [25]. Right: Distribution of deaths due to COVID-19 infection by age group and gender in Cameroon as of 23 June 2021 (after [34]).

\subsection{Volterra Integral}

The Volterra integral equation of the second kind is given as:

$$
\mathrm{S}(\mathrm{t})=\mathrm{S}(0)+\int_{0}^{\mathrm{t}} \mathcal{H}(\mathrm{t}, \epsilon) \mathrm{S}(\epsilon) \mathrm{d} \epsilon
$$

where $\mathcal{H}(t, \epsilon)$ is a kernel and $S(t)$ is the function to be solved.

\subsection{The Ross-McKendrick SIR Model}

Bernoulli [47] proposed a model for explaining the smallpox dynamics and since then, many discussions have occurred regarding the efficacy of firstly the inoculation and secondly the vaccination [48-51]. In Bernoulli's model, the population is divided into susceptibles and immunized; the probability of belonging to them is denoted as $u$ and $v$. 
The probability for a newborn individual susceptible and alive at age $\mathrm{j}$ and the probability of being immunized and alive at age $j$ is denoted as $v_{j}$. Ross [52] and McKendrick [53] (assisted later by W.O. Kermack) proposed an improvement of Bernoulli's model called the SIR model with the following equations:

$$
\frac{\mathrm{d} S}{\mathrm{dt}}=\delta \mathrm{S}+\delta \mathrm{I}+(\delta+\gamma) \mathrm{R}-\beta \mathrm{SI}-\mu \mathrm{S}, \frac{\mathrm{dI}}{\mathrm{dt}}=\beta \mathrm{SI}-(\mu+\nu) \mathrm{I}, \frac{\mathrm{dR}}{\mathrm{dt}}=v \mathrm{I}-(\mu+\gamma) \mathrm{R}
$$

where $S$ is the size of the susceptible, I the size of the infectious, and R the size of the recovered with the total population $\mathrm{N}$ defined as $\mathrm{N}=\mathrm{S}+\mathrm{I}+\mathrm{R}$. $\beta$ is the contagion rate, $\delta$ the birth rate (supposed to be equal to the death rate), $\gamma$ is the loss of immune resistance, and $v$ is the immunization rate. The basic reproduction number $R_{0}=\frac{\beta N}{v+\delta}$ is the mean number of individuals secondarily infectious by one primary infectious. $R_{0}$ predicts, if it is greater than 1, the occurrence of an epidemic wave. By defining age classes between 1 and $m$ and by denoting $S_{j}, I_{j}, R_{j}, j=1,2, \ldots, m$, each subpopulation of $S, I$, and $R$ at age $j$, we can define at any stationary state $\left(S^{*}, I^{*}, R^{*}\right)$ the probabilities for a newborn individual of being alive and either susceptible, infectious or immune at age $\mathrm{j}$ by the following formula:

$$
\mathrm{u}^{*}(\mathrm{j})=\frac{\mathrm{S}_{\mathrm{j}}^{*}}{\mathrm{P}^{*}}, \mathrm{v}^{*}(\mathrm{j})=\frac{\mathrm{I}_{\mathrm{j}}^{*}}{\mathrm{P}^{*}}, \mathrm{w}^{*}(\mathrm{j})=\frac{\mathrm{R}_{\mathrm{j}}^{*}}{\mathrm{P}^{*}}, \text { where } \mathrm{P} *=\Sigma_{\mathrm{i}=1, \mathrm{~m}}\left(\mathrm{~S}_{\mathrm{i}}+\mathrm{I}_{\mathrm{i}}+\mathrm{R}_{\mathrm{i}}\right)
$$

That makes the link between the Bernoulli and the Ross and Kermack-McKendrick models, but the weakness of the latter still resides in many insufficiencies and approximations:

- When the population size of either susceptible or infectious populations tends to be very large, the quadratic term SI has to be replaced by a Michealian saturation term SI $\overline{(\mathrm{k}+\mathrm{S})(\mathrm{k} /+\mathrm{I})}$

- the immunized infectious or healthy carriers are neglected in the absence of real information on their quantity and their influence on the spread of COVID-19;

- the total population size is supposed to be constant, the birth rate equaling the natural mortality. The Bernoulli model [47] takes implicitly into account the birth rate, and explicitly the natural mortality. The model by d'Alembert $[54,55]$ improved Bernoulli's model by distinguishing the specific mortality due to the infectious disease from the natural one, being more widely applicable than the model by Bernoulli which was restricted to immunizing infections. In the $\mathrm{d}^{\prime}$ Alembert method, the only task was to calculate the survival function after eliminating the particular cause of death due to the infectious disease, but Bernoulli's approach provided much more insight for a mechanistic interpretation of infectious disease real data;

- $\quad$ variables and parameters do not depend on space, i.e., neither migration nor population displacement;

- $\quad$ parameters do not depend on time which means no genetic adaptation of an infectious agent or a human population, even if it is very slow compared to the fast dynamics of epidemics.

Our proposed model is an improvement of the Ross and Kermack-McKendrick model by trying to compensate for a part of these defects. We will first introduce two age classes to account for adults and the elderly in the population and then take account of vaccination before applying our model to some countries chosen as examples.

\section{New SIGR Model Formulation}

We propose a Susceptible-Infectious-Goneanewsusceptible-Recovered (SIGR) model as an improvement of the Ross and Kermack-McKendrick models including age class and vaccination state for COVID-19 in a given population. Neglecting differences between kids and young adults, we only retain two age classes: adult and elderly, 1 and 2 . We assume that all infectious are symptomatic and we consider birth and natural death rates $\beta$ and $\mu$, as well as specific fatality rate $\epsilon$ due to the disease. Age groups $(i=1,2)$ concern individuals susceptible, infectious $I_{i}$, gone anew susceptible $G_{i}$, and a fully recovered and 
resistant $R_{i}$. We denote for each age group $(i=1,2)$ the transmission rates $\varphi_{i}$, fertility, and loss of resistance rates $\beta_{\mathrm{ij}}$ (supposed to be equal inside an age class, for the sake of simplicity), natural death rates $\mu_{2}{ }^{\mathrm{S}}, \mu_{2}{ }^{\mathrm{I}}, \mu_{2}{ }^{\mathrm{G}}$ and $\mu_{2}{ }^{\mathrm{R}}$, vaccination rates group $\theta_{i}^{\mathrm{G}}$ and $\theta_{i}^{\mathrm{R}}$, survival rates from age 1 to age $2 \mathrm{a}_{1}^{\mathrm{S}}, \mathrm{a}_{1}^{\mathrm{I}}, \mathrm{a}_{1}^{\mathrm{G}}$ and $\mathrm{a}_{1}^{\mathrm{R}}$, specific death rate due to the disease $\epsilon_{i}$, relapsed rate $\eta_{i}$ and recovery rate $\gamma_{i}$ (cf. Table 1$)$.

Table 1. List of the parameters considered in the SIGR (Susceptible-Infectious-GoneanewsusceptibleRecovered) model.

\begin{tabular}{|c|c|}
\hline \multicolumn{2}{|c|}{ Epidemiologic Parameters } \\
\hline $\mathrm{S}_{\mathrm{i}}$ & $\begin{array}{l}\begin{array}{l}\text { Susceptible individuals of age class i among age classes, adult } 1 \text { and } \\
\text { elderly } 2 .\end{array}\end{array}$ \\
\hline $\mathrm{I}_{\mathrm{i}}$ & Infectious individuals of age class $\mathrm{i}$ \\
\hline $\mathrm{G}_{\mathrm{i}}$ & Gone anew susceptible individuals of age class i \\
\hline $\mathrm{R}_{\mathrm{i}}$ & Fully recovered and resistant individuals of age class i \\
\hline $\mathrm{R}_{\mathrm{o}}$ & Basic reproduction number \\
\hline$\phi_{\mathrm{i}}$ & Transmission rate of age class $i$ \\
\hline$\eta_{\mathrm{i}}$ & Relapsed rate of age class i \\
\hline$\gamma_{\mathrm{i}}$ & Recovering rate of age class $i$ \\
\hline $\mathrm{a}_{1}{ }^{\mathrm{K}}$ & Survival rate from age 1 to age 2 for compartment $K$ of age class 1 \\
\hline$\mu_{2}{ }^{K}$ & Natural death rate of compartment $K$ of age class 2 \\
\hline \multicolumn{2}{|c|}{ Demographic Parameters } \\
\hline$\beta_{\mathrm{i} 1}$ & Birth and loss of resistance of recovered from compartments of age class $\mathrm{i}$ \\
\hline$\beta_{22}$ & Loss of resistance of recovered rate from compartments of age class 2 \\
\hline$\theta_{\mathrm{i}}^{\mathrm{K}}$ & Vaccination rate from compartment $\mathrm{K}$ of age class $\mathrm{i}$ \\
\hline$\epsilon_{\mathrm{i}}$ & Specific fatality rate $\epsilon$ due to the disease of age class $i$ \\
\hline
\end{tabular}

The description above can be illustrated by the following set of non-linear differential Equation (1), while the graphical representation of the model is given in Figure 5:

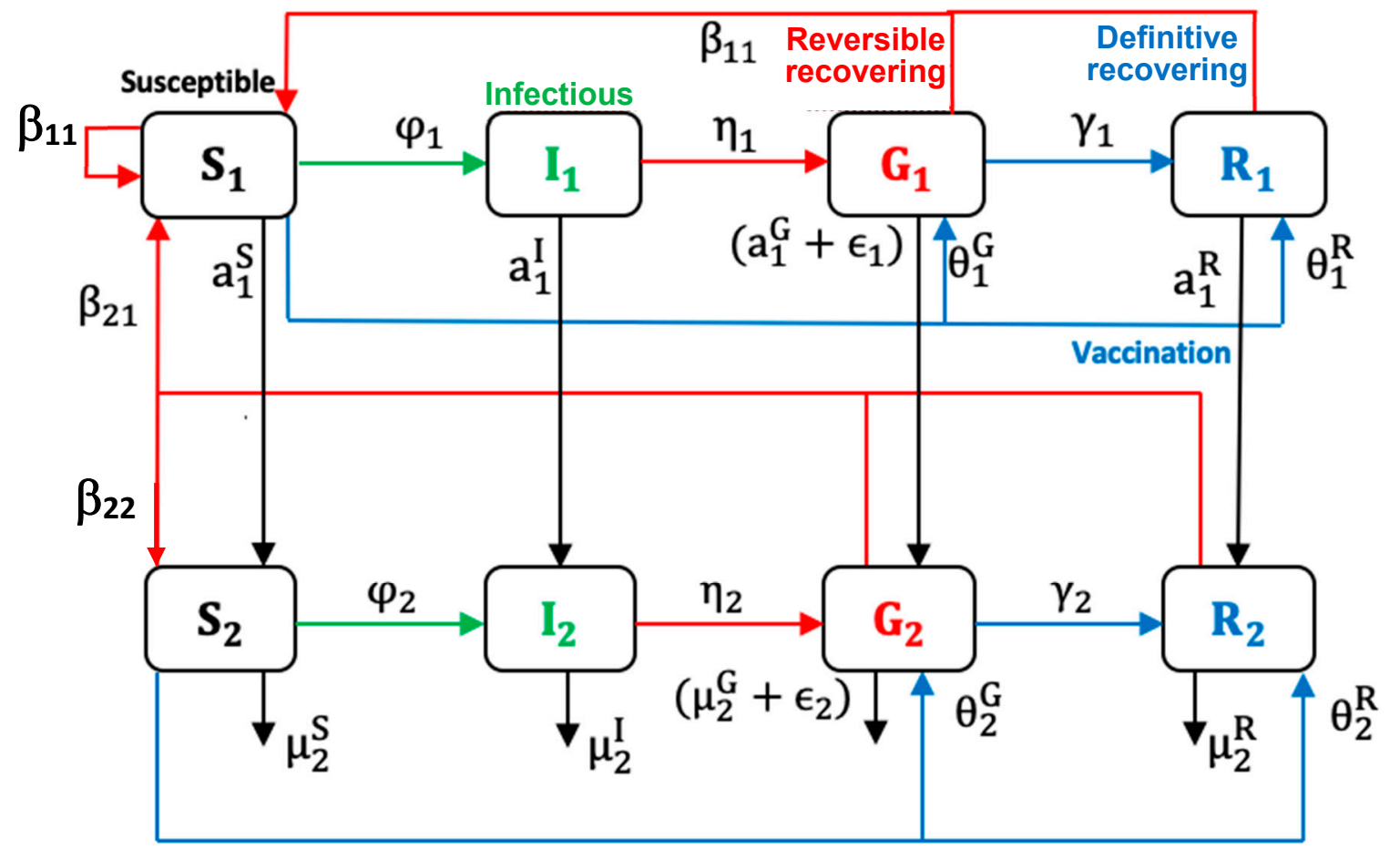

Figure 5. Age-dependent scheme for COVID-19 outbreak modeling. 


$$
\begin{gathered}
\mathrm{dS}_{1} / \mathrm{dt}=\beta_{11}\left(\mathrm{~S}_{1}+\mathrm{G}_{1}+\mathrm{R}_{1}\right)+\beta_{21}\left(\mathrm{~S}_{2}+\mathrm{G}_{2}+\mathrm{R}_{2}\right)-\left(\mathrm{a}_{1}^{\mathrm{S}}+\theta_{1}^{\mathrm{G}}+\theta_{1}^{\mathrm{R}}+\varphi_{1}\left(\mathrm{I}_{1}+\mathrm{I}_{2}\right)\right) \mathrm{S}_{1} \\
\mathrm{dS}_{2} / \mathrm{dt}=\mathrm{a}_{1}^{\mathrm{S}} \mathrm{S}_{1}-\mu_{2} \mathrm{~S}_{2}-\left(\theta_{2}^{\mathrm{G}}+\theta_{2}^{\mathrm{R}}+\varphi_{2}\left(\mathrm{I}_{1}+\mathrm{I}_{2}\right)\right) \mathrm{S} 2+\beta_{22}\left(\mathrm{G}_{2}+\mathrm{R}_{2}\right) \\
\mathrm{dI} \mathrm{I}_{1} / \mathrm{dt}=\varphi_{1}\left(\mathrm{I}_{1}+\mathrm{I}_{2}\right) \mathrm{S}_{1}-\left(\mathrm{a}_{1}^{\mathrm{I}}+\eta_{1}\right) \mathrm{I}_{1} \\
\mathrm{dI}_{2} / \mathrm{dt}=\varphi_{2}\left(\mathrm{I}_{1}+\mathrm{I}_{2}\right) \mathrm{S}_{2}+\mathrm{a}_{1}^{\mathrm{I}} \mathrm{I}_{1}-\eta_{2} \mathrm{I}_{2}-\mu_{2} \mathrm{I}_{2} \\
\mathrm{dG}_{1} / \mathrm{dt}=\eta_{1} \mathrm{I}_{1}+\theta_{1}^{\mathrm{G}} \mathrm{S}_{1}-\left(\mathrm{a}_{1}^{\mathrm{G}}+\gamma_{1}+\epsilon_{1}\right) \mathrm{G}_{1} \\
\mathrm{dG}_{2} / \mathrm{dt}=\eta_{2} \mathrm{I}_{2}+\mathrm{a}_{1}^{\mathrm{G}} \mathrm{G}_{1}+\theta_{2}^{\mathrm{G}} \mathrm{S}_{2}-\left(\gamma_{2}+\mu_{2}^{\mathrm{G}}+\epsilon_{2}+\beta_{22}\right) \mathrm{G}_{2} \\
\mathrm{dR}_{1} / \mathrm{dt}=\gamma_{1} \mathrm{G}_{1}+\theta_{1}^{\mathrm{R}} \mathrm{S}_{1}-\mathrm{a}_{1}^{\mathrm{R}} \mathrm{R}_{1} \\
\mathrm{dR}_{2} / \mathrm{dt}=\gamma_{2} \mathrm{G}_{2}+\theta_{2}^{\mathrm{R}} \mathrm{S}_{2}+\mathrm{a}_{1}^{\mathrm{R}} \mathrm{R}_{1}-\left(\mu_{2}{ }^{\mathrm{R}}+\beta_{22}\right) \mathrm{R}_{2}
\end{gathered}
$$

with $S_{1}(t) \geq 0, S_{2}(t) \geq 0, I_{1}(t) \geq 0, I_{2}(t) \geq 0, G_{1}(t) \geq 0, G_{2}(t) \geq 0, R_{1}(t) \geq 0, R_{2}(t) \geq 0$. We will suppose in the following that the loss of resistance rates is neglectable.

\section{Mathematical Analysis of the Model}

\subsection{Positivity of the Solution}

Lemma 1. Let the initial condition be given as follows:

$\left\{S_{1}(0), S_{2}(0), I_{1}(0), I_{2}(0), G_{1}(0), G_{2}(0), R_{1}(0), R_{2}(0) \geq 0\right\}$, then solutions of system of Equation (1) are positive for all $t>0$.

Proof From the first equation in the model Equation (1), we obtain:

$$
\mathrm{dS}_{1} / \mathrm{dt}=\beta_{11}\left(\mathrm{~S}_{1}+\mathrm{G}_{1}+\mathrm{R}_{1}\right)+\beta_{21}\left(\mathrm{~S}_{2}+\mathrm{G}_{2}+\mathrm{R}_{2}\right)-\left(\mathrm{a}_{1}^{\mathrm{S}}+\theta_{1}^{\mathrm{G}}+\theta_{1}^{\mathrm{R}}+\varphi_{1}\left(\mathrm{I}_{1}+\mathrm{I}_{2}\right)\right) \mathrm{S}_{1},
$$

Hence, $\mathrm{dS}_{1} / \mathrm{dt} \geq\left(\beta_{11}-\mathrm{a}_{1}^{\mathrm{S}}-\theta_{1}^{\mathrm{G}}-\theta_{1}^{\mathrm{R}}\right) \mathrm{S}_{1}$

By using the separating variable method and then, integrating, we obtain:

$$
\begin{gathered}
\int \frac{d S_{1} / d t}{S_{1}} \geq \int\left(\beta_{11}-a_{1}^{S}-\theta_{1}^{G}-\theta_{1}^{R}\right) d t \\
\ln S_{1} \geq\left(\beta_{11}-a_{1}^{S}-\theta_{1}^{G}-\theta_{1}^{R}\right) t+k
\end{gathered}
$$

Finally, by writing $S_{1}(0)=e^{k}$, we have: $S_{1}(t) \geq S_{1}(0) e^{\left(\beta_{11}-a_{1}^{S}-\theta_{1}^{G}-\theta_{1}^{R}\right) t} \geq 0$.

By applying the same process to other equations in (1), we have:

$$
\begin{gathered}
S_{2}(t) \geq S_{2}(0) e^{\left(a_{1}^{S}-\theta_{2}^{G}-\theta_{2}^{R}-\mu_{2}{ }^{S}\right) t} \geq 0 \\
I_{1}(t) \geq I_{1}(0) e^{-\left(a_{1}^{I}+\eta_{1}\right) t} \geq 0 \\
I_{2}(t) \geq I_{2}(0) e^{-\left(\eta_{2} I_{2}+\mu_{2}{ }^{I}\right) t} \geq 0 \\
G_{1}(t) \geq G_{1}(0) e^{-\left(a_{1}^{G}+\gamma_{1}+\epsilon_{1}\right) t} \geq 0 \\
G_{2}(t) \geq G_{2}(0) e^{-\left(\gamma_{2}+\mu_{2}{ }^{G}+\epsilon_{2}\right) t} \geq 0 \\
R_{1}(t) \geq R_{1}(0) e^{-a_{1}^{R} t} \geq 0 \\
R_{2}(t) \geq R_{2}(0) e^{-\mu_{2} R^{R}} \geq 0
\end{gathered}
$$

Then, the solutions of Equation (1) $\left\{\mathrm{S}_{1}(\mathrm{t}), \mathrm{S}_{2}(\mathrm{t}), \mathrm{I}_{1}(\mathrm{t}), \mathrm{I}_{2}(\mathrm{t}), \mathrm{G}_{1}(\mathrm{t}), \mathrm{G}_{2}(\mathrm{t}), \mathrm{R}_{1}(\mathrm{t}), \mathrm{R}_{2}(\mathrm{t})\right\}$ are positive for all $\mathrm{t}>0$. 


\subsection{Boundedness of the Solution}

Let denote by $\mathrm{S}$ the total size of all individuals:

$$
S(t)=S_{1}(t)+S_{2}(t)+I_{1}(t)+I_{2}(t)+G_{1}(t)+G_{2}(t)+R_{1}(t)+R_{2}(t)
$$

Then, by adding all the model Equations (1), we have:

$\mathrm{dS} / \mathrm{dt}=\beta_{11}\left(\mathrm{~S}_{1}+\mathrm{G}_{1}+\mathrm{R}_{1}\right)+\beta_{21}\left(\mathrm{~S}_{2}+\mathrm{G}_{2}+\mathrm{R}_{2}\right)-\left(\mu_{2}{ }^{\mathrm{S}} \mathrm{S}_{2}+\mu_{2}{ }^{\mathrm{I}} \mathrm{I}_{2}+\mu_{2}{ }^{\mathrm{G}} \mathrm{G}_{2}+\epsilon \mathrm{G}_{2}+\mu_{2}{ }^{\mathrm{R}} \mathrm{R}_{2}\right.$

Let us denote $\mu=\inf \left\{\mu_{2}{ }^{S}, \mu_{2}{ }^{I}, \mu_{2}{ }^{G}+\epsilon, \mu_{2}{ }^{R}\right\}$. By neglecting the fecundity rate of the young class and if $\beta=\beta_{21} \leq \mu$, we have:

$\mathrm{dS} / \mathrm{dt} \leq \beta\left(\mathrm{S}_{2}+\mathrm{I}_{2}+\mathrm{G}_{2}+\mathrm{R}_{2}\right)-\mu\left(\mathrm{S}_{2}+\mathrm{I}_{2}+\mathrm{G}_{2}+\mathrm{R}_{2}\right) \leq 0$,

Then, we can conclude that the total size $S$ is bounded, which implies the boundedness of the partial sizes $S_{1}(t), S_{2}(t), I_{1}(t), I_{2}(t), G_{1}(t), G_{2}(t), R_{1}(t), R_{2}(t)$.

\subsection{Disease-Free (Eradication) Equilibrium}

Setting the right hand side of the model equations (1) to zero, i.e.,

$$
\mathrm{dS}_{1} / \mathrm{dt}=\mathrm{dS}_{2} / \mathrm{dt}=\mathrm{dI}_{1} / \mathrm{dt}=\mathrm{dI}_{2} / \mathrm{dt}=\mathrm{G}_{1} / \mathrm{dt}=\mathrm{dG}_{2} / \mathrm{dt}=\mathrm{dR}_{1} / \mathrm{dt}=\mathrm{dR}_{2} / \mathrm{dt}=0
$$

and supposing that all infectious class sizes are equal to zero which means that there is no disease eradication in the studied population, thus the disease-free equilibrium state is:

$$
\left(S_{1}{ }^{*}, S_{2}{ }^{*}, I_{1}{ }^{*}, I_{2}{ }^{*} G_{1}{ }^{*}, G_{2}{ }^{*}, R_{1}{ }^{*}, R_{2}{ }^{*}\right)=\left\{\frac{\beta_{21} S_{2}}{a_{1}^{S}+\theta_{1}^{\mathrm{G}}+\theta_{1}^{R}-\beta_{11}}, \frac{a_{1}^{S} S_{1}}{\mu_{2}{ }^{S}+\theta_{2}^{G}+\theta_{2}^{R}}, 0,0,0,0,0,0\right\}
$$

\subsection{Proof of Stability of the Endemic State}

Let us fix a set of values for the model parameters, where $\mathrm{k}$ is a scale parameter:

$$
\begin{gathered}
\varphi_{1}=\varphi_{2}=\frac{4 \mathrm{k}^{2}}{100}, \mu_{2}^{S}=\epsilon_{1}=\beta_{22}=0, \mu_{2}^{I}=\frac{499 \mathrm{k}}{100}, \mu_{2}{ }^{\mathrm{G}}=\frac{49 \mathrm{k}}{100}, \mu_{2}{ }^{\mathrm{R}}=\frac{2 \mathrm{k}}{5}, \beta_{11}=\beta_{21}=\frac{\mathrm{k}}{5}, \\
\theta_{1}^{\mathrm{G}}=\theta_{1}^{\mathrm{R}}=\gamma_{1}=\gamma_{2}=0.1 \mathrm{k}, \theta_{2}^{\mathrm{G}}=\theta_{2}^{\mathrm{R}}=\mathrm{k}, \mathrm{a}_{1}^{\mathrm{S}}=\mathrm{a}_{1}^{\mathrm{I}}=\mathrm{a}_{1}^{\mathrm{G}}=\mathrm{a}_{1}^{\mathrm{R}}=\frac{98 \mathrm{k}}{96}, \epsilon_{2}=\frac{4 \mathrm{k}}{5}, \eta_{1}=\eta_{2}=0.2 \mathrm{k}
\end{gathered}
$$

The two stationary points are labeled with ${ }^{*}$ (resp. $\left.{ }^{* *}\right)$ for the eradication (resp. endemic) state:

$$
\left(S_{1}{ }^{*}, S_{2}{ }^{*}, I_{1}{ }^{*}, I_{2}{ }^{*} G_{1}{ }^{*}, G_{2}{ }^{*}, R_{1}{ }^{*}, R_{2}{ }^{*}\right)=\left(\frac{\beta_{21} S_{2}}{a_{1}^{S}+\theta_{1}^{\mathrm{G}}+\theta_{1}^{R}-\beta_{11}}, \frac{a_{1}^{S} S_{1}}{\mu_{2}{ }^{S}+\theta_{2}^{G}+\theta_{2}^{R}}, 0,0,0,0,0,0\right)
$$

and

$$
\left(\mathrm{S}_{1}{ }^{* *}, \mathrm{~S}_{2}{ }^{* *}, \mathrm{I}_{1}{ }^{* *}, \mathrm{I}^{* *}, \mathrm{G}_{1}{ }^{* *}, \mathrm{G}_{2}{ }^{* *}, \mathrm{R}_{1}{ }^{* *}, \mathrm{R}_{2}{ }^{* *}\right)=(10,10,10,10,20,40,15,15) \text {, if } \mathrm{k}=1 .
$$

Let us show that the endemic state is locally stable. With the chosen parameter values and $\mathrm{k}=1$, from the model Equation (1), we have:

$$
\begin{gathered}
\mathrm{dS}_{1} / \mathrm{dt}=0.2 \mathrm{G}_{1}-1.02 \mathrm{~S}_{1}+0.2 \mathrm{R}_{1}+0.2 \mathrm{~S}_{2}+0.2 \mathrm{G}_{2}+0.2 \mathrm{R}_{2}-0.04 \mathrm{I}_{1} \mathrm{~S}_{1}-0.04 \mathrm{I}_{2} \mathrm{~S}_{1} \\
\mathrm{dS}_{2} / \mathrm{dt}=1.02 \mathrm{~S}_{1}-2 \mathrm{~S}_{2}-0.04 \mathrm{I}_{1} \mathrm{~S}_{2}-0.04 \mathrm{I}_{2} \mathrm{~S}_{2} \\
\mathrm{dI}_{1} / \mathrm{dt}=0.04 \mathrm{I}_{1} \mathrm{~S}_{1}+0.04 \mathrm{I}_{2} \mathrm{~S}_{1}-1.04 \mathrm{I}_{1} \\
\mathrm{dI}_{2} / \mathrm{dt}=0.04 \mathrm{I}_{1} \mathrm{~S}_{2}+0.04 \mathrm{I}_{2} \mathrm{~S}_{2}+1.02 \mathrm{I}_{1}-5.19 \mathrm{I}_{2} \\
\mathrm{dG}_{1} / \mathrm{dt}=0.2 \mathrm{I}_{1}+0.1 \mathrm{~S}_{1}-1.12 \mathrm{G}_{1} \\
\mathrm{dG}_{2} / \mathrm{dt}=0.2 \mathrm{I}_{2}+1.02 \mathrm{G}_{1}+\mathrm{S}_{2}-1.39 \mathrm{G}_{2}
\end{gathered}
$$




$$
\begin{gathered}
\mathrm{dR}_{1} / \mathrm{dt}=0.1 \mathrm{G}_{1}+0.1 \mathrm{~S}_{1}-1.02 \mathrm{R}_{1} \\
\mathrm{dR}_{2} / \mathrm{dt}=0.1 \mathrm{G}_{2}+\mathrm{S}_{2}+1.02 \mathrm{R}_{1}-0.4 \mathrm{R}_{2}
\end{gathered}
$$

By calculating the Jacobian matrix $\mathrm{M}$ of the system of Equation (2) at the endemic state, where I is the identity matrix and finding the roots of its characteristic polynomial $\mathrm{P}_{\mathrm{M}}$, we have for the second stationary point the expression as follows:

$$
\mathrm{M}-\lambda \mathrm{I}=\left(\begin{array}{cccccccc}
-1.02-\lambda & 0.2 & -0.4 & -0.4 & 0.2 & 0.2 & 0.2 & 0.2 \\
1.02 & -2-\lambda & -0.4 & -0.4 & 0 & 0 & 0 & 0 \\
0.8 & 0 & -1.04-\lambda & 0 & 0 & 0 & 0 & 0 \\
0 & 0.8 & 1.02 & -5.19-\lambda & 0 & 0 & 0 & 0 \\
0.1 & 0 & 0.2 & 0 & -1.12-\lambda & 0 & 0 & 0 \\
0 & 1 & 0 & 0.2 & 1.02 & -1.39-\lambda & 0 & 0 \\
0.1 & 0 & 0 & 0 & 0.1 & 0 & -1.02-\lambda & 0 \\
0 & 1 & 0 & 0 & 0 & 0.1 & 1.02 & -0.4-\lambda
\end{array}\right)
$$

The roots of the characteristic polynomial of $\mathrm{M}, \mathrm{P}_{\mathrm{M}}$, satisfy:

$$
\begin{gathered}
\mathrm{P}_{\mathrm{M}}(\lambda)=\operatorname{det}(\mathrm{M}-\lambda \mathrm{I})=(\lambda+0.706923)(\lambda+5.13103)\left(\lambda^{2}+0.973528 \lambda+0.34052\right) \\
\left(\lambda^{2}+2.0371 \lambda+1.03861\right)\left(\lambda^{2}+3.69142 \lambda+3.53083\right)=0
\end{gathered}
$$

The real parts of the eigenvalues of the matrix $\mathrm{M}$ are all negative, equal to:

$-0706923,-5.13103,-0.486764,-1.01855,-1.84571$

Hence, with the set of chosen parameter values, the stability of the endemic state is proved.

\subsection{Existence and Unicity of the Solution}

We want to establish existence and unicity of the solution for the model Equation (1). Let us denote the second member of (1) by $\mathcal{H}=\left(\mathcal{H}_{1}, \mathcal{H}_{2}, \mathcal{H}_{3}, \mathcal{H}_{4}, \mathcal{H}_{5}, \mathcal{H}_{6}, \mathcal{H}_{7}, \mathcal{H}_{8}\right)$ and the state vector by $Z=\left(S_{1}, S_{2}, I_{1}, I_{2}, G_{1}, G_{2}, R_{1}, R_{2}\right)$, with $Z(0)=Z_{0}, Z(t, \epsilon)=\mathcal{H}(t, \epsilon) Z(t)$. The Volterra integral equation formulation of the model Equation (1) is the following:

$$
\begin{aligned}
\mathrm{S}_{1}(\mathrm{t}) & =\mathrm{S}_{1}(0)+\int_{0}^{\mathrm{t}} \mathcal{H}_{1}(\mathrm{t}, \epsilon) \mathrm{S}_{1}(\epsilon) \mathrm{d} \epsilon \\
\mathrm{S}_{2}(\mathrm{t}) & =\mathrm{S}_{2}(0)+\int_{0}^{\mathrm{t}} \mathcal{H}_{2}(\mathrm{t}, \epsilon) \mathrm{S}_{2}(\epsilon) \mathrm{d} \epsilon \\
\mathrm{I}_{1}(\mathrm{t}) & =\mathrm{I}_{1}(0)+\int_{0}^{\mathrm{t}} \mathcal{H}_{3}(\mathrm{t}, \epsilon) \mathrm{I}_{1}(\epsilon) \mathrm{d} \epsilon \\
\mathrm{I}_{2}(\mathrm{t}) & =\mathrm{I}_{2}(0)+\int_{0}^{\mathrm{t}} \mathcal{H}_{4}(\mathrm{t}, \epsilon) \mathrm{I}_{2}(\epsilon) \mathrm{d} \epsilon \\
\mathrm{G}_{1}(\mathrm{t}) & =\mathrm{G}_{1}(0)+\int_{0}^{\mathrm{t}} \mathcal{H}_{5}(\mathrm{t}, \epsilon) \mathrm{G}_{1}(\epsilon) \mathrm{d} \epsilon \\
\mathrm{G}_{2}(\mathrm{t}) & =\mathrm{G}_{2}(0)+\int_{0}^{\mathrm{t}} \mathcal{H}_{6}(\mathrm{t}, \epsilon) \mathrm{G}_{2}(\epsilon) \mathrm{d} \epsilon \\
\mathrm{R}_{1}(\mathrm{t}) & =\mathrm{R}_{1}(0)+\int_{0}^{\mathrm{t}} \mathcal{H}_{7}(\mathrm{t}, \epsilon) \mathrm{R}_{1}(\epsilon) \mathrm{d} \epsilon \\
\mathrm{R}_{2}(\mathrm{t}) & =\mathrm{R}_{2}(0)+\int_{0}^{\mathrm{t}} \mathcal{H}_{8}(\mathrm{t}, \epsilon) \mathrm{R}_{2}(\epsilon) \mathrm{d} \epsilon
\end{aligned}
$$

Let $\mathcal{S}_{1}$ be the solution for $\mathrm{S}_{1}$ and kernels $\mathcal{H}_{\mathrm{I}}, \mathrm{i}=1,2, \ldots, 8$ satisfy Lipschitz conditions: $\sup _{0<\mathrm{t} \leq 1}\left\|S_{1}\right\| \leq \mathrm{c}_{1}, \sup _{0<\mathrm{t} \leq 1}\left\|\mathrm{~S}_{2}\right\| \leq \mathrm{c}_{2}, \sup _{0<\mathrm{t} \leq 1}\left\|\mathrm{I}_{1}\right\| \mathrm{c}_{3}, \sup _{0<\mathrm{t} \leq 1}\left\|\mathrm{I}_{2}\right\| \mathrm{c}_{4}, \sup _{0<\mathrm{t} \leq 1}\left\|\mathrm{G}_{1}\right\| \leq$ $c_{5}, \sup _{0<t<1}\left\|G_{2}\right\| \leq c_{6}, \sup _{0<t \leq 1}\left\|R_{1}\right\| \leq c_{7}, \sup _{0<t \leq 1}\left\|R_{2}\right\| \leq c_{8}$ and $c_{i}>0$, for $\mathrm{i}=1,2, \ldots, 8$. Then, following inequalities hold, using triangle inequality and properties of the $\mathcal{H}_{\mathrm{i}}^{\prime}$ s norm: 


$$
\begin{gathered}
\left\|\mathcal{H}_{1}\left(\mathrm{~S}_{1}\right)-\mathcal{H}_{1}\left(\mathcal{S}_{1}\right)\right\| \leq\left[\beta_{11}-\right. \\
\left.-\left(\mathrm{a}_{1}^{\mathrm{S}}+\theta_{1}^{\mathrm{G}}+\theta_{1}^{\mathrm{R}}+\varphi_{1}\left(\mathrm{I}_{1}+\mathrm{I}_{2}\right)\right)\right] \mathrm{S}_{1}-\left[\beta_{11}-\left(\mathrm{a}_{1}^{\mathrm{S}}+\theta_{1}^{\mathrm{G}}+\theta_{1}^{\mathrm{R}}+\varphi_{1}\left(\mathrm{I}_{1}+\mathrm{I}_{2}\right)\right)\right] \mathcal{S}_{1} \| \\
\leq\left\|\beta_{11}-\left(\mathrm{a}_{1}^{\mathrm{S}}+\theta_{1}^{\mathrm{G}}+\theta_{1}^{\mathrm{R}}+\varphi_{1}\left(\mathrm{I}_{1}+\mathrm{I}_{2}\right)\right)\right\|\left\|\mathrm{S}_{1}-\mathcal{S}_{1}\right\| \\
\leq\left\|\beta_{11}-\left(\mathrm{a}_{1}^{\mathrm{S}}+\theta_{1}^{\mathrm{G}}+\theta_{1}^{\mathrm{R}}+\varphi_{1} \mathrm{c}_{3}+\varphi_{1} \mathrm{c}_{4}\right)\right\|\left\|\mathrm{S}_{1}-\mathcal{S}_{1}\right\| \\
=\partial_{1}\left\|\mathrm{~S}_{1}-\mathcal{S}_{1}\right\|
\end{gathered}
$$

where $\xi=\beta_{11}\left(G_{1}+R_{1}\right)+\beta_{21}\left(S_{2}+G_{2}+R_{2}\right), \partial_{1}=\beta_{11}-a_{1}^{S}-\theta_{1}^{G}-\theta_{1}^{R}-\varphi_{1} c_{3}-\varphi_{1} c_{4}$.

Therefore, $\mathcal{H}_{1}$ satisfies the Lipschitz conditions. We can show in the same way that other functions $\mathcal{H}_{\mathrm{i}}, \mathrm{i}=2, \ldots, 8$ in the model Equation (2) satisfy the Lipschitz conditions as follows:

$$
\begin{aligned}
\| \mathcal{H}_{2}\left(\mathrm{~S}_{2}\right)-\mathcal{H}_{2}\left(\mathcal{S}_{2}\right) & \leq\left\|\partial_{2}\right\| \mathrm{S}_{2}-\mathcal{S}_{2} \| \\
\| \mathcal{H}_{3}\left(\mathrm{I}_{1}\right)-\mathcal{H}_{3}\left(\mathcal{L}_{1}\right) & \leq\left\|\partial_{3}\right\| \mathrm{I}_{1}-\mathcal{L}_{1} \| \\
\| \mathcal{H}_{4}\left(\mathrm{I}_{2}\right)-\mathcal{H}_{4}\left(\mathcal{L}_{2}\right) & \leq\left\|\partial_{4}\right\| \mathrm{I}_{2}-\mathcal{L}_{2} \| \\
\| \mathcal{H}_{5}\left(\mathrm{G}_{1}\right)-\mathcal{H}_{5}\left(\mathcal{G}_{1}\right) & \leq\left\|\partial_{5}\right\| \mathrm{G}_{1}-\mathcal{G}_{1} \| \\
\| \mathcal{H}_{6}\left(\mathrm{G}_{2}\right)-\mathcal{H}_{6}\left(\mathcal{G}_{2}\right) & \leq\left\|\partial_{6}\right\| \mathrm{G}_{2}-\mathcal{G}_{2} \| \\
\| \mathcal{H}_{7}\left(\mathrm{R}_{1}\right)-\mathcal{H}_{7}\left(\Re_{1}\right) & \leq\left\|\partial_{7}\right\| \mathrm{R}_{1}-\Re_{1} \| \\
\| \mathcal{H}_{8}\left(\mathrm{R}_{2}\right)-\mathcal{H}_{8}\left(\Re_{2}\right) & \leq\left\|\partial_{8}\right\| \mathrm{R}_{2}-\Re_{2} \|
\end{aligned}
$$

Let us now consider the following Neumann series:

$$
\begin{aligned}
\mathrm{S}_{1 \mathrm{~m}}(\mathrm{t}) & =\mathrm{S}_{1}(0)+\int_{0}^{\mathrm{t}} \mathcal{H}_{1}(\mathrm{t}, \epsilon) \mathrm{S}_{1 \mathrm{~m}-1}(\epsilon) \mathrm{d} \epsilon \\
\mathrm{S}_{2 \mathrm{~m}}(\mathrm{t}) & =\mathrm{S}_{2}(0)+\int_{0}^{\mathrm{t}} \mathcal{H}_{2}(\mathrm{t}, \epsilon) \mathrm{S}_{2 \mathrm{~m}-1}(\epsilon) \mathrm{d} \epsilon \\
\mathrm{I}_{1 \mathrm{~m}}(\mathrm{t}) & =\mathrm{I}_{1}(0)+\int_{0}^{\mathrm{t}} \mathcal{H}_{3}(\mathrm{t}, \epsilon) \mathrm{I}_{1 \mathrm{~m}-1}(\epsilon) \mathrm{d} \epsilon \\
\mathrm{I}_{2 \mathrm{~m}}(\mathrm{t}) & =\mathrm{I}_{2}(0)+\int_{0}^{\mathrm{t}} \mathcal{H}_{4}(\mathrm{t}, \epsilon) \mathrm{I}_{2 \mathrm{~m}-1}(\epsilon) \mathrm{d} \epsilon \\
\mathrm{G}_{1 \mathrm{~m}}(\mathrm{t}) & =\mathrm{G}_{1}(0)+\int_{0}^{\mathrm{t}} \mathcal{H}_{5}(\mathrm{t}, \epsilon) \mathrm{G}_{1 \mathrm{~m}-1}(\epsilon) \mathrm{d} \epsilon \\
\mathrm{G}_{2 \mathrm{~m}}(\mathrm{t}) & =\mathrm{G}_{2}(0)+\int_{0}^{\mathrm{t}} \mathcal{H}_{6}(\mathrm{t}, \epsilon) \mathrm{G}_{2 \mathrm{~m}-1}(\epsilon) \mathrm{d} \epsilon \\
\mathrm{R}_{1 \mathrm{~m}}(\mathrm{t}) & =\mathrm{R}_{1}(0)+\int_{0}^{\mathrm{t}} \mathcal{H}_{7}(\mathrm{t}, \epsilon) \mathrm{R}_{1 \mathrm{~m}-1}(\epsilon) \mathrm{d} \epsilon \\
\mathrm{R}_{2 \mathrm{~m}}(\mathrm{t}) & =\mathrm{R}_{2}(0)+\int_{0}^{\mathrm{t}} \mathcal{H}_{8}(\mathrm{t}, \epsilon) \mathrm{R}_{2 \mathrm{~m}-1}(\epsilon) \mathrm{d} \epsilon
\end{aligned}
$$

These Neumann series are convergent due to the Lipschtizian character of $\mathcal{H}^{\prime} \mathrm{s}$, then:

$$
\begin{aligned}
& \left\|S_{1 \mathrm{~m}+1}-S_{1 \mathrm{~m}}\right\| \leq \int_{0}^{\mathrm{t}}\left\|\mathcal{H}_{1}(\mathrm{t}, \epsilon) \mathrm{S}_{1 \mathrm{~m}}(\epsilon)-\mathcal{H}_{1}(\mathrm{t}, \epsilon), \mathrm{S}_{1 \mathrm{~m}-1}(\epsilon) \mathrm{d} \epsilon\right\| \\
& \leq \int_{0}^{\mathrm{t}}\left\|\mathrm{S}_{1 \mathrm{~m}}(\mathrm{t})-\mathrm{S}_{1 \mathrm{~m}-1}(\mathrm{t})\right\| \mathrm{d} \epsilon \leq \partial_{1}\left\|S_{1 \mathrm{~m}}(\mathrm{t})-\mathrm{S}_{1 \mathrm{~m}-1}(\mathrm{t})\right\|_{\infty}
\end{aligned}
$$


Other equations are given as follows:

$$
\begin{aligned}
& \left\|S_{2 m+1}-S_{2 m}\right\| \leq \partial_{2}\left\|S_{2 m}(t)-S_{2 m-1}(t)\right\|_{\infty} \\
& \left\|I_{1 m+1}-I_{1 m}\right\| \leq \partial_{3}\left\|I_{1 m}(t)-I_{1 m-1}(t)\right\|_{\infty} \\
& \left\|I_{2 m+1}-I_{2 m}\right\| \leq \partial_{4}\left\|I_{2 m}(t)-I_{2 m-1}(t)\right\|_{\infty} \\
& \left\|G_{1 m+1}-G_{1 m}\right\| \leq \partial_{5}\left\|G_{1 m}(t)-G_{1 m-1}(t)\right\|_{\infty} \\
& \left\|G_{2 m+1}-G_{2 m}\right\| \leq \partial_{6}\left\|G_{2 m}(t)-G_{2 m-1}(t)\right\|_{\infty} \\
& \left\|R_{1 m+1}-R_{1 m}\right\| \leq \partial_{7}\left\|R_{1 m}(t)-R_{1 m-1}(t)\right\|_{\infty} \\
& \left\|R_{2 m+1}-R_{2 m}\right\| \leq \partial_{8}\left\|R_{2 m}(t)-R_{2 m-1}(t)\right\|_{\infty}
\end{aligned}
$$

The above inequalities prove the existence of the function $\mathcal{H}$. We now show the uniqueness of the solution by assuming that the kernels $\mathcal{H}_{\mathrm{i}}, \mathrm{i}=1,2, \ldots, 8$ are separable, i.e., $\mathcal{H}_{1}(\mathrm{t}, \epsilon)=\varnothing(\mathrm{t}) \sigma(\epsilon)$. By denoting:

$$
\zeta(\mathrm{t})=\int_{0}^{\mathrm{t}} \mathrm{S}_{1}(\epsilon) \sigma(\epsilon) \mathrm{d} \epsilon
$$

then, because $S_{1}(t)=S_{1}(0)+\int_{0}^{t} \mathcal{H}_{1}(t, \epsilon) S_{1}(\epsilon) d \epsilon=S_{1}(0)+\int_{0}^{t} \varnothing(t) \sigma(\epsilon) S_{1}(\epsilon) d \epsilon$, we have:

$$
\zeta^{\prime}(\mathrm{t})=\varnothing(\mathrm{t}) \sigma(\mathrm{t}) \zeta(\mathrm{t})+\mathrm{S}_{1}(0) \sigma(\mathrm{t})
$$

If $\sigma(0)=0$, the solution $\zeta(\mathrm{t})$ follows $\zeta(\mathrm{t})=\mathrm{e}^{\int_{0}^{\mathrm{t}} \varnothing(\epsilon) \sigma(\epsilon) \mathrm{d} \epsilon}$ and from the definition of $\zeta(t)$, the solution of $S_{1}(t)$ is given as:

$$
\mathrm{S}_{1}(\mathrm{t})=\mathrm{S}_{1}(0)+\varnothing(\mathrm{t}) \int_{0}^{\mathrm{t}} \mathrm{S}_{1}(\epsilon) \sigma(\epsilon) \mathrm{d} \epsilon=\mathrm{S}_{1}(0)+\varnothing(\mathrm{t}) \zeta(\mathrm{t})=\mathrm{S}_{1}(0)+\varnothing(\mathrm{t}) \mathrm{e}^{\int_{0}^{\mathrm{t}} \varnothing(\epsilon) \sigma(\epsilon) \mathrm{d} \epsilon}
$$

Hence, by the unicity of the solution $\zeta(t)$, there exists just only one continuous solution for $S_{1}(t)$. Following the same approach, we can obtain a unique solution for the remaining equations of the system (2).

\subsection{Basic Reproduction Number}

In this section, we apply the idea of a next-generation matrix by linearizing the model Equation (1) near the endemic state, for the infectious part of the system (1), and we obtain:

$$
\begin{gathered}
\mathrm{dI}_{1} / \mathrm{dt}=\varphi_{1}\left(\mathrm{I}_{1}+\mathrm{I}_{2}\right) \mathrm{S}_{1}{ }^{* *}-\left(\mathrm{a}_{1}^{\mathrm{I}}+\eta_{1}\right) \mathrm{I}_{1}, \\
\mathrm{dI}_{2} / \mathrm{dt}=\varphi_{2}\left(\mathrm{I}_{1}+\mathrm{I}_{2}\right) \mathrm{S}_{2}{ }^{* *}+\mathrm{a}_{1}^{\mathrm{I}} \mathrm{I}_{1}-\eta_{2} \mathrm{I}_{2}-\mu_{2} \mathrm{I}_{2}, \\
\mathrm{dG}_{1} / \mathrm{dt}=\eta_{1} \mathrm{I}_{1}+\theta_{1}^{\mathrm{G}} \mathrm{S}_{1}{ }^{* *}-\left(\mathrm{a}_{1}^{\mathrm{G}}+\gamma_{1}+\epsilon_{1}\right) \mathrm{G}_{1}, \\
\mathrm{dG}_{2} / \mathrm{dt}=\eta_{2} \mathrm{I}_{2}+\mathrm{a}_{1}^{\mathrm{G}} \mathrm{G}_{1}+\theta_{2}^{\mathrm{G}} \mathrm{S}_{2}{ }^{* *}-\left(\gamma_{2}+\mu_{2}{ }^{\mathrm{G}}+\epsilon_{2}+\beta_{22}\right) \mathrm{G}_{2}
\end{gathered}
$$

By summing Equation (4) and by denoting I as the size of all infectious, we have:

$\mathrm{I}(\mathrm{t})=\mathrm{I}_{1}(\mathrm{t})+\mathrm{I}_{2}(\mathrm{t})+\mathrm{G}_{1}(\mathrm{t})+\mathrm{G}_{2}(\mathrm{t})$, and

$\mathrm{dI} / \mathrm{dt}=\varphi_{1}\left(\mathrm{I}_{1}+\mathrm{I}_{2}\right) \mathrm{S}_{1}{ }^{* *}+\varphi_{2}\left(\mathrm{I}_{1}+\mathrm{I}_{2}\right) \mathrm{S}_{2}{ }^{* *}-\mu_{2}{ }^{\mathrm{I}} \mathrm{I}_{2}+\theta_{1}^{\mathrm{G}} \mathrm{S}_{1}{ }^{* *}-\left(\gamma_{1}+\epsilon_{1}\right) \mathrm{G}_{1}-\left(\gamma_{2}+\right.$ $\left.\epsilon_{2}+\beta_{22}\right) G_{2}$

The matrix $J$ of the linearized system near a state $\left(S_{1}, S_{2}\right)$ is:

$$
J=\left[\begin{array}{cccc}
\varphi_{1} S_{1}-a_{1}^{I}-\eta_{1} & \varphi_{1} S_{1} & 0 & 0 \\
\varphi_{2} S_{2}+a_{1}^{I} & \varphi_{2} S_{2}-\eta_{2}-\mu_{2}{ }^{I} & 0 & 0 \\
\eta_{1} & 0 & -a_{1}^{G}-\gamma_{1}-\epsilon_{1} & 0 \\
0 & \eta_{2} & a_{1}^{G} & -\gamma_{2}-\mu_{2}^{G}-\epsilon_{2}-\beta_{22}
\end{array}\right]
$$

The corresponding characteristic polynomial $\mathrm{P}_{\mathrm{J}}(\lambda)$ is equal to: 


$$
\begin{gathered}
\left(\varphi_{1} S_{1}-a_{1}^{I}-\eta_{1}-\lambda\right)\left(\varphi_{2} S_{2}-\eta_{2}-\mu_{2}{ }^{I}-\lambda\right)\left(-a_{1}^{G}-\gamma_{1}-\epsilon_{1}-\lambda\right)\left(-\mu_{2}^{G}-\gamma_{2}-\epsilon_{2}-\beta_{22}-\lambda\right) \\
-\varphi_{1} S_{1}\left(\varphi_{2} S_{2}+a_{1}^{I}\right)\left(-a_{1}^{G}-\gamma_{1}-\epsilon_{1}-\lambda\right)\left(-\mu_{2}^{G}-\gamma_{2}-\epsilon_{2}-\beta_{22}-\lambda\right)=P_{J}(\lambda)
\end{gathered}
$$

The positive eigenvalues of $\mathrm{J}$ are roots of the following polynomial:

$$
\lambda^{2}-\left(\varphi_{1} S_{1}-a_{1}^{I}-\eta_{1}+\varphi_{2} S_{2}-\eta_{2}-\mu_{2}^{I}\right) \lambda-\varphi_{1} S_{1}\left(\varphi_{2} S_{2}+a_{1}^{I}\right)+\left(\varphi_{1} S_{1}-a_{1}^{I}-\eta_{1}\right)\left(\varphi_{2} S_{2}-\eta_{2}-\mu_{2}^{I}\right)
$$

These roots are equal to: $B \pm\left(B^{2}-C\right)^{1 / 2}$, where $B$ and $C$ are equal to:

$$
\begin{gathered}
B=\frac{\varphi_{1} S_{1}-a_{1}^{\mathrm{I}}-\eta_{1}+\varphi_{2} S_{2}-\eta_{2}-\mu_{2}{ }^{\mathrm{I}}}{2} \\
C=-\varphi_{1} S_{1}\left(\varphi_{2} S_{2}+a_{1}^{\mathrm{I}}\right)+\left(\varphi_{1} S_{1}-\mathrm{a}_{1}^{\mathrm{I}}-\eta_{1}\right)\left(\varphi_{2} S_{2}-\eta_{2}-\mu_{2}{ }^{\mathrm{I}}\right)
\end{gathered}
$$

Then, we have:

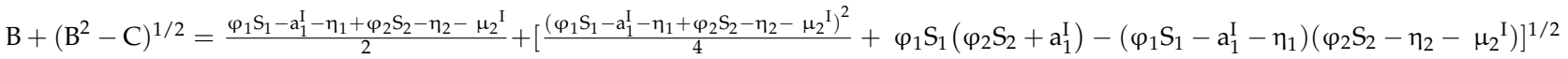

Hence, near unstable endemic state the positive dominant eigenvalue $\Lambda$ is equal to:

$$
\Lambda=\frac{\varphi_{1} S_{1}-a_{1}^{\mathrm{I}}-\eta_{1}+\varphi_{2} S_{2}-\eta_{2}-\mu_{2}{ }^{\mathrm{I}}}{2}+\left[\frac{\left(\varphi_{1} S_{1}-\mathrm{a}_{1}^{\mathrm{I}}-\eta_{1}-\varphi_{2} \mathrm{~S}_{2}+\eta_{2}+\mu_{2}^{\mathrm{I}}\right)^{2}}{4}+\varphi_{1} \mathrm{~S}_{1}\left(\varphi_{2} \mathrm{~S}_{2}+\mathrm{a}_{1}^{\mathrm{I}}\right)\right]^{1 / 2}
$$

Therefore, the basic reproduction number $\mathrm{R}_{0}$ equal to $\Lambda$ near the endemic stationary state depends mainly on the infection rates $\varphi_{1}$ and $\varphi_{2}$, when the sizes $S_{1}$ and $S_{2}$ are sufficiently important. If after a change of parameter values, the endemic state becomes unstable and $\mathrm{R}_{0}$ is becoming more than 1 , then an epidemic wave starts.

\section{Numerical Simulation of the Model: Some Examples of the COVID-19 Outbreak in Kuwait, France and Cameroon}

First, we provide some explanations for the data used for the simulations, including how we assumed some of the parameters, calculated others, and selected some from the literature cited in this article. In order to determine the susceptible classes sizes, we used Wikipedia data on the three studied countries to obtain their total population size. We then calculated the ratio between the young and elderly from the data presented in Figures 2-4 and used this ratio to determine the sizes value for the two susceptible classes at the exponential phase considered (for Kuwait 28 December 2020, France 30 October 2021, and Cameroon 19 September 2021), because the Ross and Kermack-McKendrick model is only suited for the exponential growth phase of an epidemic wave. Because the progression rates to the classes reversed recovery and fully recovered in Figure 5 are not zero, we assumed that they should have some populations at the start of the wave, even if they were small. The transmission rate for Cameroon was chosen at the start of the second wave in January 2021 from [56,57], for France at the start of the fifth wave in December 2021 [58], and for Kuwait at the start of the fourth wave in December 2021 [59]. The values of specific death rates due to disease and natural death rates for Kuwait and France were taken from [58-60]. For Cameroon, the natural death rate was taken from [61], while the specific death rate due to disease was calculated from the cumulated deaths number due to the disease in two years divided by the cumulated infectious number in these two years [61]. The vaccination rate was chosen from [62], while the loss of resistance was chosen from [63]. Other parameters were assumed.

\subsection{COVID-19 Outbreak in Kuwait}

We have chosen the following set of parameter values corresponding to the COVID-19 outbreak at the start of the fourth wave in December 2021 in Kuwait: 


$$
\begin{gathered}
\varphi_{1}=1.7, \varphi_{2}=0.9, \mu_{2}{ }^{\mathrm{S}}=0.003, \epsilon_{1}=0.28, \beta_{22}=0, \mu_{2}{ }^{\mathrm{I}}=0.0025, \mu_{2}{ }^{\mathrm{G}}= \\
0.002, \mu_{2}{ }^{\mathrm{R}}=0.0021, \beta_{11}=2.1, \beta_{21}=2.3, \theta_{1}^{\mathrm{G}}=0.765, \theta_{1}^{\mathrm{R}}=0.678, \gamma_{1}= \\
0.62, \gamma_{2}=0.74, \theta_{2}^{\mathrm{G}}=0.45, \theta_{2}^{\mathrm{R}}=0.33, \mathrm{a}_{1}^{\mathrm{S}}=0.7, \mathrm{a}_{1}^{\mathrm{I}}=0.54, \mathrm{a}_{1}^{\mathrm{G}}=0.65, \mathrm{a}_{1}^{\mathrm{R}}= \\
0.8, \epsilon_{2}=0.19, \eta_{1}=0.3 \text { and } \eta_{2}=0.38 .
\end{gathered}
$$

with initial values for $S_{1}=4413099, S_{2}=51422, I_{1}=65, I_{2}=194, G_{1}=20, G_{2}=17, R_{1}=30$ and $\mathrm{R}_{2}=73$.

We present the visualization results for the simulated values in Figure 6.
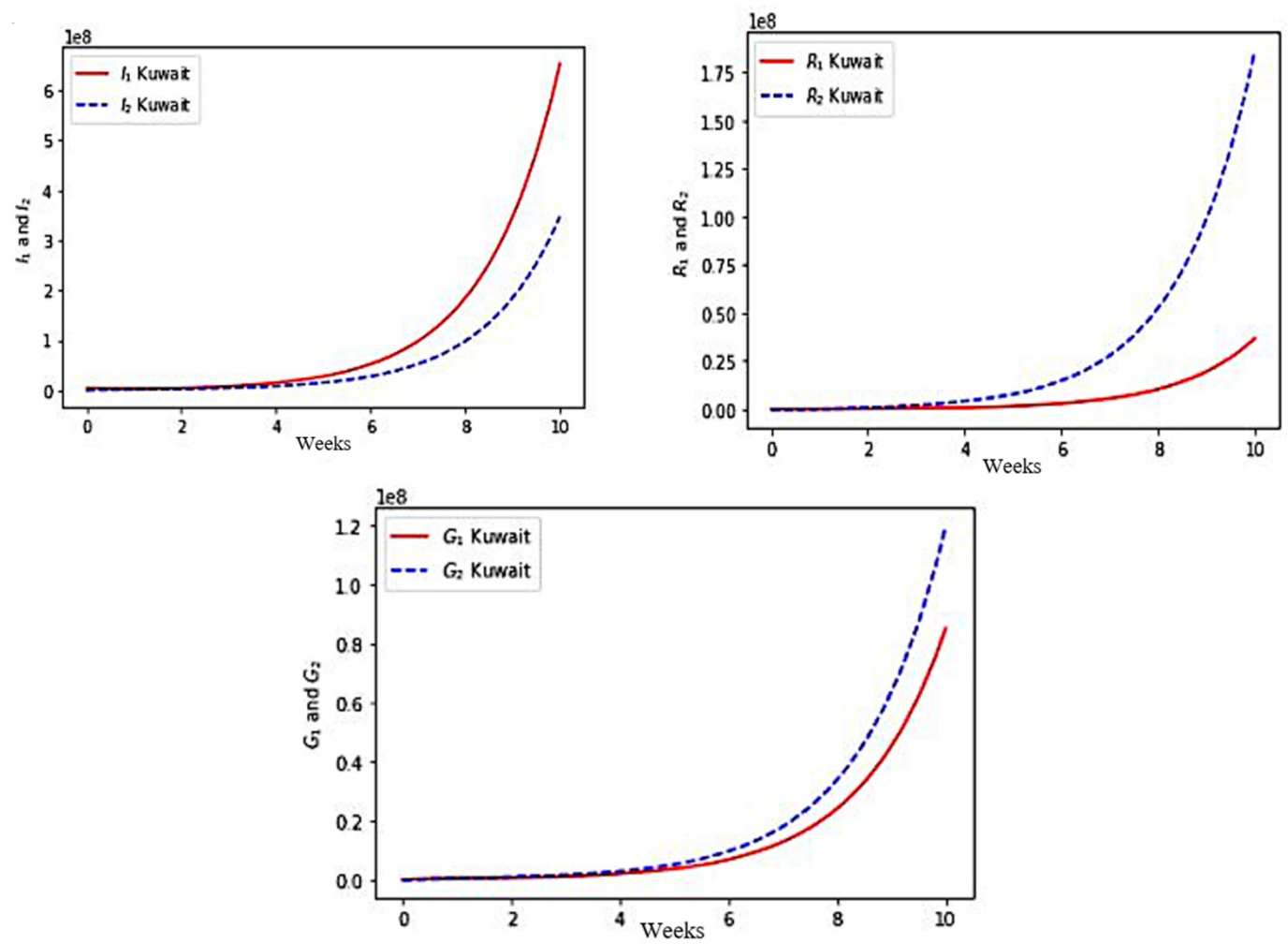

Figure 6. Numerical simulation of the variables $I_{1}$ and $I_{2}, R_{1}$ and $R_{2}, G_{1}$ and $G_{2}$ for Kuwait.

\subsection{COVID-19 Outbreak in France}

We have chosen the following set of parameter values corresponding to the COVID-19 outbreak at the start of the fifth wave in December 2021 in France:

$$
\begin{aligned}
& \varphi_{1}=1.2, \varphi_{2}=0.9, \mu_{2}{ }^{\mathrm{S}}=0.009, \epsilon_{1}=0.28, \beta_{22}=0, \mu_{2}{ }^{\mathrm{I}}=0.0025, \mu_{2}{ }^{\mathrm{G}}= \\
& 0.002, \mu_{2}{ }^{\mathrm{R}}=0.0021, \beta_{11}=1.9, \beta_{21}=2.2, \theta_{1}^{\mathrm{G}}=0.735, \theta_{1}^{\mathrm{R}}=0.678, \gamma_{1}= \\
& 0.62, \gamma_{2}=0.74, \theta_{2}^{\mathrm{G}}=0.45, \theta_{2}^{\mathrm{R}}=0.33, \mathrm{a}_{1}^{\mathrm{S}}=0.7, \mathrm{a}_{1}^{\mathrm{I}}=0.54, \mathrm{a}_{1}^{\mathrm{G}}=0.65, \mathrm{a}_{1}^{\mathrm{R}}= \\
& 0.8, \epsilon_{2}=0.19, \eta_{1}=0.3 \text { and } \eta_{2}=0.38 .
\end{aligned}
$$

with initial values for $S_{1}=53372880, S_{2}=14017120, I_{1}=3912, I_{2}=1757, G_{1}=200, G_{2}=170$, $\mathrm{R}_{1}=300$ and $\mathrm{R}_{2}=730$.

We present the visualization results for the simulated values in Figure 7. 

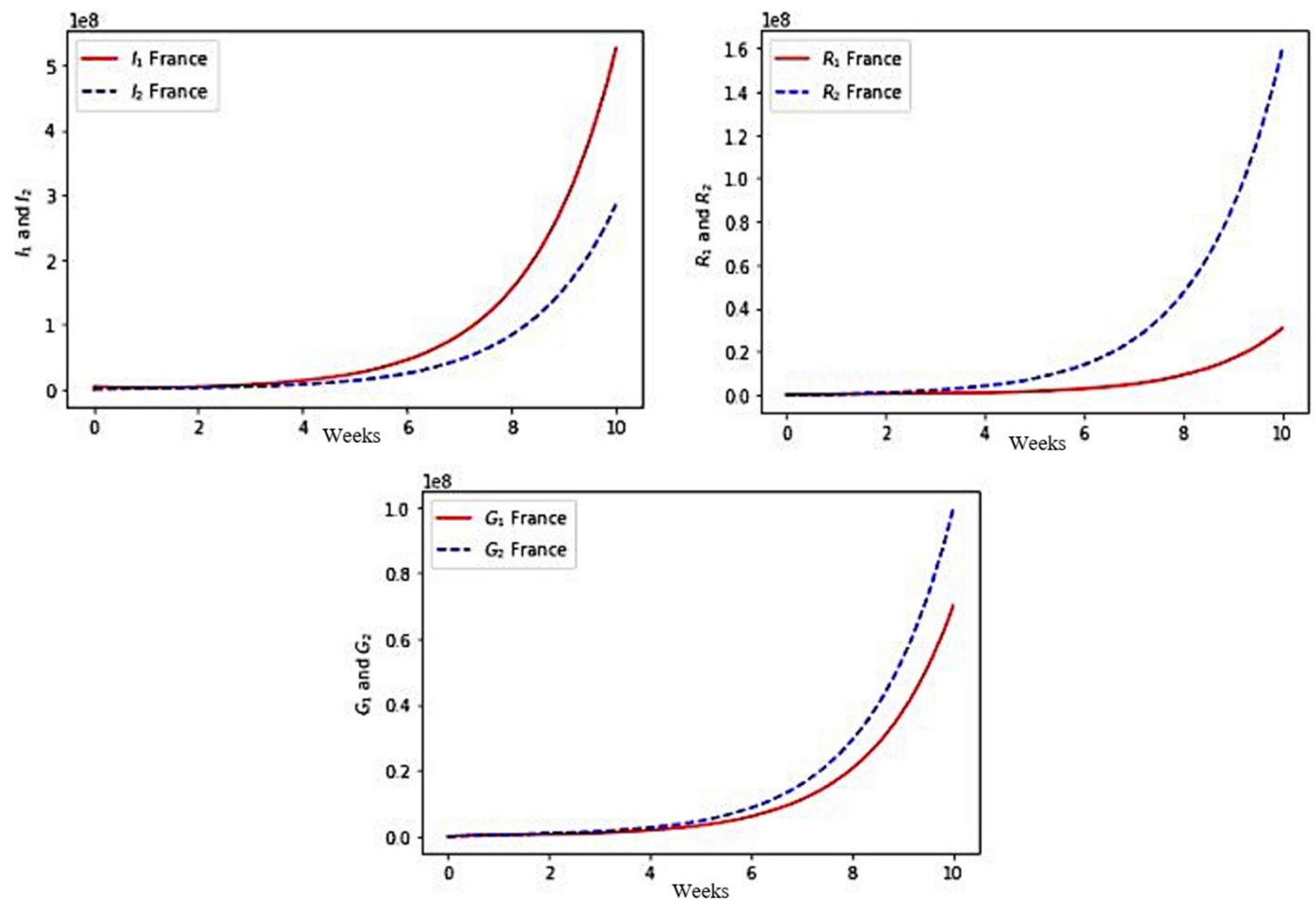

Figure 7. Numerical simulation of the variables $I_{1}$ and $I_{2}, R_{1}$ and $R_{2}, G_{1}$ and $G_{2}$ for France.

\subsection{COVID-19 Outbreak in Cameroon}

We choose the following set of parameter values corresponding to the COVID-19 outbreak at the start of the second wave in January 2021 in Cameroon:

$$
\begin{gathered}
\varphi_{1}=1.3, \varphi_{2}=0.9, \mu_{2}{ }^{\mathrm{S}}=0.009, \epsilon_{1}=0.28, \beta_{22}=0, \mu_{2}{ }^{\mathrm{I}}=0.0025, \mu_{2}^{\mathrm{G}}= \\
0.002, \mu_{2}{ }^{\mathrm{R}}=0.0021, \beta_{11}=4.5, \beta_{21}=4.1, \theta_{1}^{\mathrm{G}}=0.024, \theta_{1}^{\mathrm{R}}=0.678, \gamma_{1}= \\
0.62, \gamma_{2}=0.74, \theta_{2}^{\mathrm{G}}=0.45, \theta_{2}^{\mathrm{R}}=0.33, \mathrm{a}_{1}^{\mathrm{S}}=0.7, \mathrm{a}_{1}^{\mathrm{I}}=0.54, \mathrm{a}_{1}^{\mathrm{G}}=0.65, \mathrm{a}_{1}^{\mathrm{R}}= \\
0.8, \epsilon_{2}=0.19, \eta_{1}=0.3 \text { and } \eta_{2}=0.38 .
\end{gathered}
$$

with initial values for $S_{1}=25828973, S_{2}=721027, I_{1}=151, I_{2}=21, G_{1}=75, G_{2}=33, R_{1}=56$ and $R_{2}=64$.

We present the visualization results for the simulated values in Figure 8.
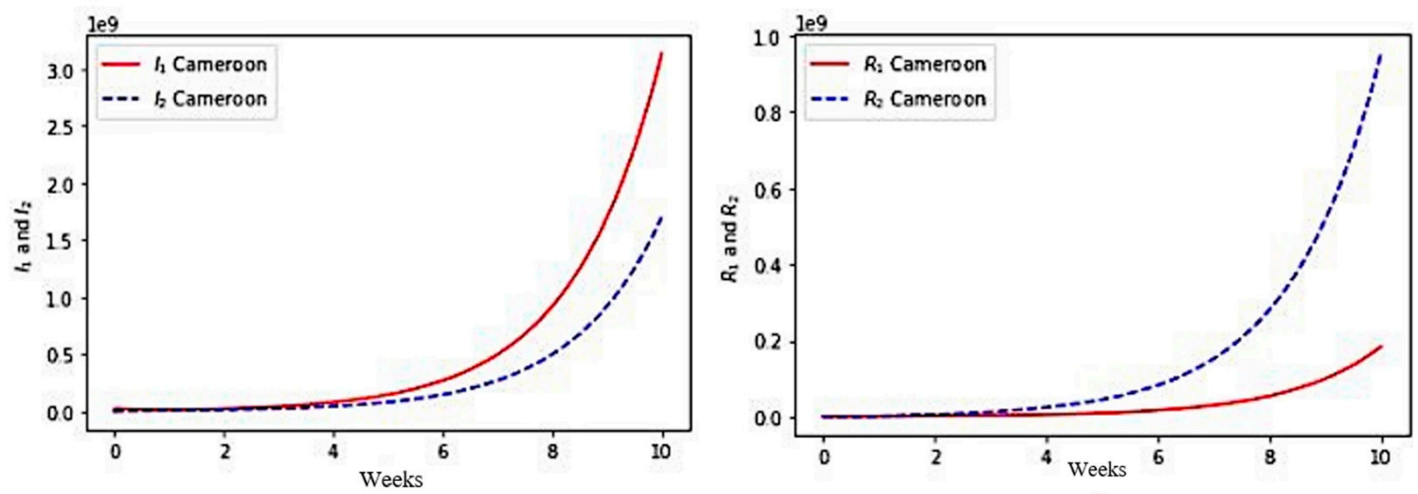

Figure 8. Cont. 


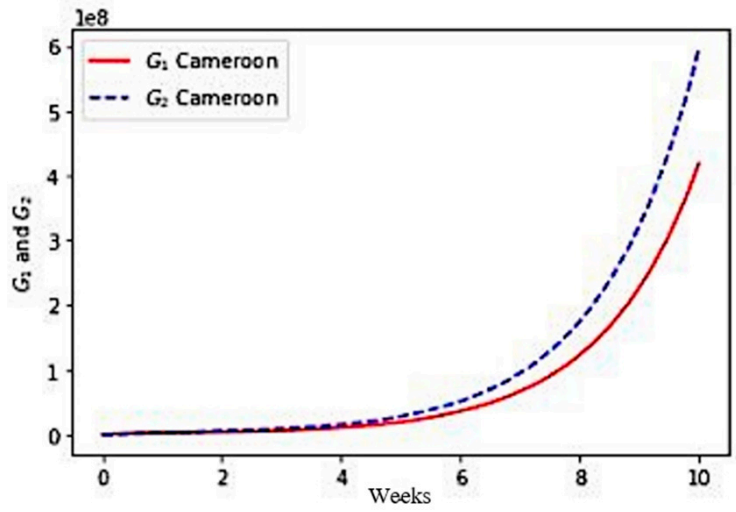

Figure 8. Numerical simulation of the variables $I_{1}$ and $I_{2}, R_{1}$ and $R_{2}, G_{1}, G_{2}$ for Cameroon.

\section{Discussion}

The results for the three considered countries present some similarities but also some differences that we will discuss in the following.

First, in each case, the exponential growth of the infectious $I_{1}+I_{2}$ and completely recovering $R_{1}+R_{2}$ populations sizes correspond roughly to the data given in [57]. The model simulations give more, i.e., allows to see the part brought by each age class to the global growth.

Second, for Kuwait, Figure 6 shows a faster growth of infectious (I) in the young class ( $\leq 65$ years) than in the older class (>65 years), which is also the case for France (Figure 7 ) and for Cameroon (Figure 8). On the other hand, this phenomenon is reversed in the three countries, with regard to the growth of populations immunized in a transient manner $(\mathrm{G})$ and in a lasting manner (R). The phenomenon is more marked for Kuwait than for France, France itself having a more marked difference than for Cameroon. This is partly explained by the better vaccination rate of Kuwait than that of France and Cameroon in the young class, the effectiveness of the vaccination (which decreases in the older class) having been assumed to be equal for the three countries.

\section{Conclusions}

Taking age into account in modeling the COVID-19 pandemic makes it possible to simulate the differential dynamic behavior of the growth of infectious and immune populations, young and old, in order, for example, to adjust the vaccine policy according to the age. Future work should, for example, take into account more age groups, at least four: children (age $\leq 12$ years), adolescents (12 years $<$ age $\leq 18$ years), young adults (18 years $<$ age $\leq 65$ years), and older adults ( $>65$ years). The most important pitfall in simulations of such a model is the estimation (by observation, calculation, or assumption) of its parameters, already difficult with two age groups. A random choice of the values of the parameters in plausible intervals followed by a study of the sensitivity to the parameters of the model, could make it possible to partly overcome the constraint of parameter estimation in a more precise future epidemiological-demographic model, in particular with regard to age groups.

Another perspective would consist in introducing, thanks to Usher's model [41,42], an accelerated aging due to infection, as well as an influence of exogenous determinants such as geo-climatic, socio-economic, and health-related factors [64-76], which weight differently on the different age groups, therefore, changing the growth dynamics specific to each of the sub-populations studied in this article. These perspectives will be examined in future works. 


\begin{abstract}
Author Contributions: Conceptualization, J.D. and K.O.; methodology, J.D. and K.O.; software, K.O. and S.S.B. validation, J.D. and K.O.; formal analysis, J.D. and K.O.; investigation, J.D.; resources, J.D.; data curation, K.O., S.S.B. and J.W.; writing-original draft preparation, J.D. and K.O.; writingreview and editing, J.D. and K.O; visualization, K.O.; S.S.B. and J.W.; supervision, J.D., S.S.B.; N.M.A.-K. and M.R.; project administration, J.D., S.S.B., N.M.A.-K. and M.R. All authors have read and agreed to the published version of the manuscript.
\end{abstract}

Funding: No specific funding has been received for the research.

Acknowledgments: Ethical approval for Kuwaiti data was obtained from the Kuwait Ministry of Health's Ethical Review Committee (ERC) (No. 1529/2020).

Conflicts of Interest: The authors declare no conflict of interest.

\title{
References
}

1. Ourworldindata on Fatality. Available online: https://ourworldindata.org/grapher/case-fatality-rate-of-covid-19-vs-medianage (accessed on 10 November 2021).

2. Van Halem, K.; Bruyndonckx, R.; Van der Hilst, J.; Cox, J.; Driesen, P.; Opsomer, M.; Van Steenkiste, E.; Stessel, B.; Dubois, J.; Messiaen, P. Risk factors for mortality in hospitalized patients with COVID-19 at the start of the pandemic in Belgium: A retrospective cohort study. BMC Infect. Dis. 2020, 20, 897.

3. Nachtigall, I.; Lenga, P.; Jóźwiak, K.; Thürmann, P.; Meier-Hellmann, A.; Kuhlen, R.; Brederlau, J.; Bauer, T.; Tebbenjohanns, J.; Schwegmann, K.; et al. Clinical course and factors associated with outcomes among 1904 patients hospitalized with COVID-19 in Germany: An observational study. Clin. Microbiol. Infect. 2020, 26, 1663-1669. [CrossRef] [PubMed]

4. Ergönül, Ö.; Akyol, M.; Tanrı̈ver, C.; Tiemeier, H.; Petersen, E.; Petrosillo, N.; Gönen, M. National case fatality rates of the COVID-19 pandemic. Clin. Microbiol. Infect. 2021, 27, 118-124. [CrossRef]

5. Thiabaud, A.; Iten, A.; Balmelli, C.; Senn, L.; Troillet, N.; Widmer, A.; Flury, D.; Schreiber, P.W.; VThiabaud, A.; Iten, A.; et al. Cohort profile: SARS-CoV-2/COVID-19 hospitalized patients in Switzerland. Swiss Med. Wkly. 2021, 151, w20475. [CrossRef] [PubMed]

6. Roelens, M.; Martin, A.; Friker, B.; Sousa, F.; Thiabaud, A.; Vidondo, B.; Buchter, V.; Gardiol, C.; Vonlanthen, J.; Balmelli, C.; et al. Evolution of COVID-19 mortality over time: Result from the swiss hospital surveillance system (CH-SUR). Swiss Med. Wkly. 2021, 151, w30105.

7. Qin, W.; Bai, W.; Liu, K.; Liu, Y.; Meng, X.; Zhang, K.; Zhang, M. Clinical Course and Risk Factors of Disease Deterioration in Critically Ill Patients with COVID-19. Hum. Gene Ther. 2021, 32, 310-315. [CrossRef]

8. Surendra, H.; Elyazar, I.R.; Djaafara, B.A.; Ekawati, L.L.; Saraswati, K.; Adrian, V.; Widyastuti Oktavia, D.; Salama, N.; Lina, R.N.; Andrianto, A.; et al. Clinical characteristics and mortality associated with COVID-19 in Jakarta, Indonesia: A hospital-based retrospective cohort study. Lancet Reg. Health-West. Pac. 2021, 9, 100108. [CrossRef]

9. Odeh, M.M.; Al Qaissieh, R.; Tarifi, A.A.; Kilani, M.M.; Tadros, R.E.; Al Khashman, A.I.; Alzoubi, K.H. A prediction model of risk factors for complications among SARS-CoV2 positive patients: Cases from Jordan. J. Infect. Public Health. 2021, 14, 689-695. [CrossRef]

10. Almazeedi, S.; Al-Youha, S.; Jamal, M.H.; Al-Haddad, M.; Al-Muhaini, A.; Al-Ghimlas, F.; Al-Sabah, S. Characteristics, risk factors and outcomes among the first consecutive 1096 patients diagnosed with COVID-19 in Kuwait. eClinicalMedicine 2020, 24, 100448. [CrossRef]

11. Huang, X.; Shao, X.; Xing, L.; Hu, Y.; Sin, D.D.; Zhang, X. The impact of lockdown timing on COVID-19 transmission across US countries. eClinicalMedicine 2021, 38, 101035. [CrossRef]

12. Violato, C.; Violato, E.M.; Violato, E.M. Impact of the stringency of lockdown measures on COVID-19: A theoretical model of a pandemic. PLoS ONE 2021, 16, e0258205. [CrossRef] [PubMed]

13. Fernández-Martínez, N.F.; Ortiz-González-Serna, R.; Serrano-Ortiz, Á.; Rivera-Izquierdo, M.; Ruiz-Montero, R.; Pérez-Contreras, M.; Guerrero-Fernández de Alba, I.; Romero-Duarte, Á.; Salcedo-Leal, I. Sex Differences and Predictors of In-Hospital Mortality among Patients with COVID-19: Results from the ANCOHVID Multicentre Study. Int. J. Environ. Res. Public Health 2021, 18, 9018. [CrossRef] [PubMed]

14. Zhu, M.; Kleepbua, J.; Guan, Z.; Chew, S.P.; Tan, J.W.; Shen, J.; Latthitham, N.; Hu, J.; Law, J.X.; Li, L. Early Spatiotemporal Patterns and Population Characteristics of the COVID-19 Pandemic in Southeast Asia. Healthcare 2021, 9, 1220. [CrossRef] [PubMed]

15. Estenssoro, E.; Loudet, C.I.; Ríos, F.G.; Kanoore Edul, V.S.; Plotnikow, G.; Andrian, M.; Romero, I.; Piezny, D.; Bezzi, M.; Mandich, V.; et al. Clinical characteristics and outcomes of invasively ventilated patients with COVID-19 in Argentina (SATICOVID): A prospective, multicenter cohort study. Lancet Respir. Med. 2021, 9, 989-998. [CrossRef]

16. Martín-Sánchez, F.J.; Valls Carbó, A.; Miró, Ò.; Llorens, P.; Jiménez, S.; Piñera, P.; Burillo-Putze, G.; Martín, A.; García-Lamberechts, J.E.; Jacob, J.; et al. Socio-Demographic Health Determinants Are Associated with Poor Prognosis in Spanish Patients Hospitalized with COVID-19. J. Gen. Intern. Med. 2021, 36, 3737-3742. [CrossRef]

17. Jaspard, M.; Sow, M.S.; Juchet, S.; Dienderé, E.; Serra, B.; Kojan, R.; Sivahera, B.; Martin, C.; Kinda, M.; Lang, H.J.; et al. Clinical presentation, outcomes and factors associated with mortality: A prospective study from three COVID-19 referral care centres in West Africa. Int. J. Infect. Dis. 2021, 108, 45-52. [CrossRef]

18. Cordova, E.; Mykietiuk, A.; Sued, O.; De Vedia, L.; Pacifico, N.; Garcia Hernandez, M.H.; Baeza, N.M.; Garibaldi, F.; Alzogaray, M.F.; Contreras, R.; et al. Clinical characteristics and outcomes of hospitalized patients with SARS-CoV-2 infection in a Latin American country: Results from the ECCOVID multicenter prospective study. PLoS ONE 2021, 16, e0258260. [CrossRef] 
19. Seligmann, H.; Vuillerme, N.; Demongeot, J. Unpredictable, Counter-Intuitive Geoclimatic and Demographic Correlations of COVID-19 Spread Rates. Biology 2021, 10, 623. [CrossRef]

20. Oshinubi, K.; Rachdi, M.; Demongeot, J. Modelling of COVID-19 pandemic vis-à-vis some socio-economic factors. Front. Appl. Math. Stat. 2022, 7, 786983. [CrossRef]

21. Area, I.; Lorenzo, H.; Marcos, P.J.; Nieto, J.J. One Year of the COVID-19 Pandemic in Galicia: A Global View of Age-Group Statistics during Three Waves. Int. J. Environ. Res. Public Health 2021, 18, 5104. [CrossRef]

22. Pastorino, R.; Pezzullo, A.M.; Villani, L.; Causio, F.A.; Axfors, C.; Contopoulos-Ioannidis, D.G.; Boccia, S.; Ioannidis, J.P.A. Change in age distribution of COVID-19 deaths with the introduction of COVID-19 vaccination. Environ. Res. 2022, 204, 112342. [CrossRef] [PubMed]

23. Drees. Available online: https://data.drees.solidarites-sante.gouv.fr/explore/dataset/covid-19-resultats-par-age-issus-desappariements-entre-si-vic-si-dep-et-vac-si/analyze/ (accessed on 12 December 2021).

24. Population Pyramid. Available online: https://images.populationpyramid.net/capture/?selector=\%23pyramid-sharecontainer\&url=https $\% 3 \mathrm{~A} \% 2 \mathrm{~F} \% 2 \mathrm{Fwww}$.populationpyramid.net $\% 2 \mathrm{Ffr} \% 2 \mathrm{Ffrance} \% 2 \mathrm{~F} 2020 \% 2 \mathrm{~F} \% 3 \mathrm{Fshare} \% 3 \mathrm{Dtrue}$ (accessed on 10 November 2021).

25. Bacaër, N. A Short History of Mathematical Population Dynamics; Springer: New York, NY, USA, 2011.

26. Ndaïrou, F.; Area, I.; Nieto, J.J.; Torres, D.F.M. Mathematical modeling of COVID-19 transmission dynamics with a case study of Wuhan. Chaos Solitons Fractals 2020, 135, 109846. [CrossRef] [PubMed]

27. Demongeot, J.; Griette, Q.; Magal, P. Computations of the transmission rates in SI epidemic model applied to COVID-19 data in mainland China. R. Soc. Open Sci. 2020, 7, 201878. [CrossRef]

28. Rambo, A.P.S.; Gonçalves, L.F.; Gonzáles, A.I.; Rech, C.R.; Paiva, K.M.; Haas, P. Impact of super-spreaders on COVID-19: Systematic review. Sao Paulo Med. J. 2021, 139, 163-169. [CrossRef] [PubMed]

29. Özköse, F.; Yavuz, M. Investigation of interactions between COVID-19 and diabetes with hereditary traits using real data: A case study in turkey. Comput. Biol. Med. 2021, 141, 105044. [CrossRef]

30. Naik, P.A.; Zu, J.; Ghori, M.B.; Naik, M.U. Modeling the effects of the contaminated environments on COVID-19 transmission in India. Results Phys. 2021, 29, 104774. [CrossRef]

31. Li, M.; Zu, J.; Li, Z.; Shen, M.; Li, Y.; Ji, F. How to Reduce the Transmission Risk of COVID-19 More Effectively in New York City: An Age-Structured Model Study. Front. Med. 2021, 13, 641205. [CrossRef]

32. Naik, P.A.; Yavuz, M.; Qureshi, S.; Zu, J.; Townley, S. Modeling and analysis of COVID-19 epidemics with treatment in fractional derivatives using real data from Pakistan. Eur. Phys. J. Plus 2020, 135, 795. [CrossRef]

33. Naik, P.A.; Owolabi, K.M.; Zu, J.; Naik, M.U. Modeling the transmission dynamics of COVID-19 pandemic in Caputo type fractional derivative. J. Multiscale Model. 2021, 12, 2150006. [CrossRef]

34. Camerooon Data Report. Rapport de Situation COVID-19 n47; Ministère de la Santé du Cameroun: Yaoundé, Cameroun, 2020.

35. Demetrius, L. Boltzmann, Darwin and the directionality theory. Phys. Rep. 2013, 530, 1-86. [CrossRef]

36. Rhodes, C.J.; Demetrius, L. Evolutionary Entropy Determines Invasion Success in Emergent Epidemics. PLoS ONE 2010, 5, e12951. [CrossRef]

37. Demongeot, J.; Demetrius, L. Complexity and Stability in Biological Systems. Int. J. Bifurc. Chaos 2015, 25, 40013. [CrossRef]

38. Demetrius, L. Demographic parameters and natural selection. Proc. Natl. Acad. Sci. USA 1974, 71, 4645-4647. [CrossRef] [PubMed]

39. Demetrius, L. Statistical mechanics and population biology. J. Stat. Phys. 1983, 30, 709-750. [CrossRef]

40. Demongeot, J.; Demetrius, L. La dérive démographique et la sélection naturelle: Etude empirique de la France (1850-1965). Population 1989, 2, 231-248.

41. Demongeot, J. Biological boundaries and biological age. Acta Biotheor. 2009, 57, 397-419. [CrossRef]

42. Gaudart, J.; Ghassani, M.; Mintsa, J.; Rachdi, M.; Waku, J.; Demongeot, J. Demography and Diffusion in epidemics: Malaria and Black Death spread. Acta Biotheor. 2010, 58, 277-305. [CrossRef]

43. Demongeot, J.; Hansen, O.; Hessami, H.; Jannot, A.S.; Mintsa, J.; Rachdi, M.; Taramasco, C. Random modelling of contagious diseases. Acta Biotheor. 2013, 61, 141-172. [CrossRef]

44. Demongeot, J.; Taramasco, C. Evolution of social networks: The example of obesity. Biogerontology 2014, 15, 611-626. [CrossRef]

45. Demongeot, J.; Hansen, O.; Taramasco, C. Complex systems and contagious social diseases: Example of obesity. Virulence 2015, 7, 129-140. [CrossRef]

46. Demongeot, J.; Jelassi, M.; Taramasco, C. From Susceptibility to Frailty in social networks: The case of obesity. Math. Pop. Stud. 2017, 24, 219-245. [CrossRef]

47. Bernoulli, D. Essai d'une nouvelle analyse de la mortalité causée par la petite vérole, et des avantages de l'inoculation pour la prévenir. In Mémoire Académie Royale des Sciences; Académie Royale des Sciences: Paris, France, 1760; pp. 1-45.

48. Henderson, D.A. The eradication of smallpox-An overview of the past, present, and future. Vaccine 2011, 29, 7-9. [CrossRef] [PubMed]

49. Wujastyk, D. Medicine in India. In Oriental Medicine: An Illustrated Guide to the Asian Arts of Healing; Serindia: Chicago, IL, USA, 1995; pp. 19-37.

50. Silverstein, M. A History of Immunology, 2nd ed.; Academic Press: London, UK, 2009; p. 293.

51. Lewis, M. Lady Mary Wortley Montagu, Her Life and Letters (1689-1762); Hutchinson: London, UK, 1925.

52. Ross, R. An application of the theory of probabilities to the study of a priori pathometry. Proc. R. Soc. Ser. A 1916, 92, 204-230.

53. McKendrick, A.G. Applications of mathematics to medical problems. Proc. Edinb. Math. Soc. 1926, 44, 98-130. [CrossRef] 
54. Gaudart, J.; Touré, O.; Dessay, N.; Dicko, A.L.; Ranque, S.; Forest, L.; Demongeot, J.; Doumbo, O.K. Modelling malaria incidence with environmental dependency in a locality of Sudanese savannah area, Mali. Malar. J. 2009, 8, 61. [CrossRef]

55. D'Alembert, J. Opuscules Mathématiques; David: Paris, France, 1761; Volume 2, pp. 35-38.

56. Nkague Nkamba, L.; Manga, T.T. Modelling and Prediction of the Spread of COVID-19 in Cameroon and Assessing the Governmental Measures (March-September 2020). COVID 2021, 1, 622-644. [CrossRef]

57. Worldometers. Available online: https://www.worldometers.info/coronavirus/country/ (accessed on 10 December 2021).

58. Statista. Available online: https://fr.statista.com/statistiques/1104103/victimes-coronavirus-age-france/ (accessed on 10 December 2021).

59. Ourworldindata on Mortality. Available online: https:/ / ourworldindata.org/excess-mortality-covid (accessed on 10 December 2021).

60. Worldbank Death Rate. Available online: https://data.worldbank.org/indicator/SP.DYN.CDRT.IN (accessed on 10 December 2021).

61. Coronavirus. Available online: http:// coronavirus.politologue.com/coronavirus-Cameroun.cm (accessed on 10 December 2021)

62. Renkulab. Available online: https://renkulab.shinyapps.io/COVID-19-EpidemicForecasting/_w_cee38e19/(accessed on 10 December 2021).

63. Worldbank Fertility Rate. Available online: https:/ / data.worldbank.org/indicator/SP.DYN.TFRT.IN (accessed on 10 December 2021).

64. Oshinubi, K.; Amakor, A.; Peter, O.J.; Rachdi, M.; Demongeot, J. Approach to COVID-19 time series data using deep learning and spectral analysis methods. AIMS Bioeng. 2022, 9, 1-21. [CrossRef]

65. Oshinubi, K.; Ibrahim, F.; Rachdi, M.; Demongeot, J. Functional Data Analysis: Application to Daily Observation of COVID-19 Prevalence in France. AIMS Math. 2022, 7, 5347-5385. [CrossRef]

66. Demongeot, J.; Oshinubi, K.; Rachdi, M.; Hobbad, L.; Alahiane, M.; Iggui, S.; Gaudart, J.; Ouassou, I. The application of ARIMA model to analyse COVID-19 incidence pattern in several countries. J. Math. Comput. Sci. 2022, 12, 10.

67. Griette, Q.; Demongeot, J.; Magal, P. What can we learn from COVID-19 data by using epidemic models with unidentified infectious cases? Math. Biosci. Eng. 2022, 19, 537-594. [CrossRef]

68. Demongeot, J.; Flet-Berliac, Y.; Seligmann, H. Temperature decreases spread parameters of the new COVID-19 cases dynamics Biology 2020, 9, 94. [CrossRef] [PubMed]

69. Seligmann, H.; Iggui, S.; Rachdi, M.; Vuillerme, N.; Demongeot, J. Inverted covariate effects for mutated 2nd vs. 1st wave COVID-19: High temperature spread biased for young. Biology 2020, 9, 226. [CrossRef] [PubMed]

70. Demongeot, J.; Seligmann, H. COVID-19 and miRNA-like inhibition power. Med. Hypotheses 2020, 144, 110245. [CrossRef] [PubMed]

71. Soubeyrand, S.; Demongeot, J.; Roques, L. Towards unified and real-time analyses of outbreaks at country-level during pandemics. One Health 2020, 11, 100187. [CrossRef] [PubMed]

72. Gaudart, J.; Landier, J.; Huiart, L.; Legendre, E.; Lehot, L.; Bendiane, M.K.; Chiche, L.; Petitjean, A.; EMosnier, E.; Kirakoya-Samadoulougou, F.; et al. Factors associated with spatial heterogeneity of COVID-19 in France: A nationwide ecological study. Lancet Public Health 2021, 6, e222-e231. [CrossRef]

73. Griette, Q.; Demongeot, J.; Magal, P. A robust phenomenological approach to investigate COVID-19 data for France. Math. Appl. Sci. Eng. 2021, 2, 149-160. [CrossRef]

74. Oshinubi, K.; Rachdi, M.; Demongeot, J. Analysis of reproduction number $\mathrm{R}_{0}$ of COVID-19 using Current Health Expenditure as Gross Domestic Product percentage (CHE/GDP) across countries. Healthcare 2021, 9, 1247. [CrossRef]

75. Oshinubi, K.; Al-Awadhi, F.; Rachdi, M.; Demongeot, J. Data Analysis and Forecasting of COVID-19 Pandemic in Kuwait. Kuwait J. Sci. 2021, 9, 1-28.

76. Almalki, A.; Gokaraju, B.; Acquaah, Y.; Turlapaty, A. Regression Analysis for COVID-19 Infections and Deaths Based on Food Access and Health Issues. Healthcare 2022, 10, 324. [CrossRef] 